\title{
Impact of high energy beam tunes on the sensitivities to the standard unknowns at DUNE
}

\author{
Jogesh Rout, ${ }^{1, *}$ Samiran Roy, ${ }^{2,3,4, \dagger}$ Mehedi Masud, ${ }^{5,4, \ddagger}$ Mary Bishai, ${ }^{6, \S}$ and Poonam Mehta $\circledast^{1, \|}$ \\ ${ }^{1}$ School of Physical Sciences, Jawaharlal Nehru University, New Delhi 110067, India \\ ${ }^{2}$ Physical Research Laboratory, Navrangpura, Ahmedabad 380 009, India \\ ${ }^{3}$ Harish-Chandra Research Institute, Chattnag Road, Allahabad 211 019, India \\ ${ }^{4}$ Homi Bhabha National Institute, Training School Complex, Anushakti Nagar, Mumbai 400085, India \\ ${ }^{5}$ Institute of Physics, Sachivalaya Marg, Sainik School Post, Bhubaneswar 751005, India \\ ${ }^{6}$ Brookhaven National Laboratory, P.O. Box 5000, Upton, New York 11973, USA
}

(Received 23 September 2020; accepted 4 December 2020; published 24 December 2020)

Even though neutrino oscillations have been conclusively established, there are a few unanswered questions pertaining to leptonic charge parity violation, mass hierarchy, and $\theta_{23}$ octant degeneracy. Addressing these questions is of paramount importance at the current and future neutrino experiments including the Deep Underground Neutrino Experiment, which has a baseline of $1300 \mathrm{~km}$. In the standard mode, DUNE is expected to run with a low energy tuned beam which peaks around the first oscillation maximum (2-3 GeV) (and then sharply falls off as we go to higher energies). However, the wide band nature of the beam available at long baseline neutrino facility allows for the flexibility in utilizing beam tunes that are well suited at higher energies as well. In this work, we utilize a beam that provides high statistics at higher energies which is referred to as the medium energy beam. This opens up the possibility of exploring not only the usual oscillation channels but also the $\nu_{\mu} \rightarrow \nu_{\tau}$ oscillation channel which was otherwise not accessible. Our goal is to find an optimal combination of beam tune and runtime (with the total runtime held fixed) distributed in neutrino and antineutrino mode that leads to an improvement in the sensitivities of these parameters at the Deep Underground Neutrino Experiment. In our analysis, we incorporate all the three channels $\left(\nu_{\mu} \rightarrow \nu_{e}, \nu_{\mu} \rightarrow \nu_{\mu}, \nu_{\mu} \rightarrow \nu_{\tau}\right)$ and develop an understanding of their relative contributions in sensitivities at the level of $\Delta \chi^{2}$. Finally, we obtain the preferred combination of runtime using both the beam tunes as well as neutrino and antineutrino mode that lead to enhanced sensitivity to the current unknowns in neutrino oscillation physics, i.e., charge parity violation, mass hierarchy, and $\theta_{23}$ octant.

DOI: 10.1103/PhysRevD.102.116018

\section{INTRODUCTION}

Neutrino was first postulated by Pauli in 1930 to tackle the issue of nonconservation of energy in the $\beta$ decay spectrum and subsequently discovered experimentally in 1956 [1]. The idea of neutrino oscillations as $\nu \rightarrow \bar{\nu}$ oscillations was proposed by Pontecorvo [2,3] in 1957 which was thought to be the leptonic analog of the $K_{0} \rightarrow$ $\bar{K}_{0}$ oscillations in the hadronic sector. However, soon after

\footnotetext{
*jogesh.rout1@gmail.com

samiran@prl.res.in

\#jomiye.adda@gmail.com

\$mbishai@bnl.gov

"pm@jnu.ac.in
}

Published by the American Physical Society under the terms of the Creative Commons Attribution 4.0 International license. Further distribution of this work must maintain attribution to the author(s) and the published article's title, journal citation, and DOI. Funded by SCOAP. the discovery of the second type of neutrino $\left(\nu_{\mu}\right)$, the idea evolved into neutrino flavor oscillations which was proposed by Maki, Nakagawa, and Sakata [4] as well as by Gribov and Pontecorvo [5]. Neutrino oscillations among the three active flavors imply that at least two of the neutrino states are massive, which cannot be reconciled within the Standard Model of particle physics. The discovery of neutrino oscillations in multiple experiments involving different energies and baselines was awarded the Nobel Prize for Physics in 2015 [6]. Neutrino sector offers us with a unique opportunity to explore the mysterious world of physics beyond the Standard Model.

Neutrino oscillations are governed by six parameters: three mixing angles $\left(\theta_{12}, \theta_{13}, \theta_{23}\right)$, two mass squared differences $\left(\Delta m_{21}^{2}=m_{2}^{2}-m_{1}^{2}, \Delta m_{31}^{2}=m_{3}^{2}-m_{1}^{2}\right)$ and one Dirac $C P$ phase $(\delta)$. Among the various parameters, $\theta_{12}$, $\Delta m_{21}^{2}$ (as well as the sign of $\Delta m_{21}^{2}$ ), and $\theta_{13}$ have been measured quite precisely [7]. Also, we have a fairly good idea about the magnitude of $\Delta m_{31}^{2}$ [7]. The focus has now 
shifted to address three key questions such as what is the value of the $C P$ phase $\delta$ that enters the oscillation formalism, what is the sign of $\Delta m_{31}^{2}$ (also referred to as the neutrino mass hierarchy) and what is the octant of $\theta_{23}$ ? If $\delta$ is found to be different from 0 or $\pi$, it would imply $C P$ violation (CPV) in the leptonic sector. The answer to this question is crucially linked to a more fundamental and elusive puzzle vis-à-vis why is there baryon asymmetry in the Universe? In order to match the observed baryon asymmetry, Sakharov's conditions [8] have to be satisfied: i.e., (a) existence of Baryon number violating process, (b) $C$ and $C P$ violation, and (c) processes out of thermal equilibrium. A seminal work carried out by Fukugita was to invoke the idea of leptogenesis to achieve baryogenesis [9] (see also [10] for a review on leptogenesis). Thus, establishing whether $C P$ is violated in the leptonic sector would provide a key missing ingredient towards solving the mystery of matter-antimatter asymmetry in the observed Universe. ${ }^{1}$ The next question pertains to the determination of mass hierarchy, i.e., whether the three neutrino mass eigenstates $m_{1}, m_{2}, m_{3}$ are arranged in normal (NH, i.e., $\Delta m_{31}^{2}>0$ ) or inverted (IH i.e., $\Delta m_{31}^{2}<0$ ) hierarchy is of fundamental importance. Apart from shedding light into the plausible set of models for neutrino mass generation, ${ }^{2}$ this will also help in determining the nature of neutrino (i.e., Dirac or Majorana) through neutrinoless double beta decay via the effective Majorana mass parameter $m_{\beta \beta}$ [14-16]. Finally, the close to maximal value of the mixing angle $\theta_{23}$ could indicate the presence of a new symmetry, the $\mu-\tau$ symmetry in nature $[17,18]$. The determination of the correct octant of $\theta_{23}$, i.e., whether $\theta_{23}>\pi / 4$ (higher octant or $\mathrm{HO}$ ) or $\theta_{23}<\pi / 4$ (lower octant or $\mathrm{LO}$ ) or $\theta_{23}=\pi / 4$ (maximal mixing), plays an crucial role in validating a certain class of models to generate neutrino mass related to the $\mu-\tau$ symmetry. ${ }^{3}$

The available data from the currently running longbaseline neutrino oscillation experiments Tokai to Kamioka (T2K) [20] and NuMI Off-axis $\nu_{e}$ Appearance (NO $\nu$ A) [21] have started uncovering a few of the open issues mentioned above. Latest T2K results [22] hint towards $\mathrm{HO}$ with $\sin ^{2} \theta_{23}=0.53_{-0.04}^{+0.03}$ for both $\mathrm{NH}$ and IH. For the first time, $\mathrm{T} 2 \mathrm{~K}$ has been able to rule out a large range of values of $\delta$ around $\pi / 2$ at $3 \sigma$ C.L. irrespective of mass hierarchy. It also excludes $C P$ conservation $(\delta=0$ or $\pi)$ at $95 \%$ C.L. The most recent measurements by the $\mathrm{NO} \nu \mathrm{A}$ Collaboration [23] using both $\nu$ and $\bar{\nu}$ running mode hint towards $\mathrm{NH}$ at $1.9 \sigma$ C.L. and shows a weak preference for $\theta_{23}$ lying in $\mathrm{HO}$ at a

\footnotetext{
${ }^{1}$ See [11] for a review on leptonic CPV.

${ }^{2}$ For, e.g., for models based on flavor symmetry, the ones exhibiting softly broken $L_{e}-L_{\mu}-L_{\tau}$ symmetry predict IH [12] and GUT models employing a type I seesaw mechanism prefers $\mathrm{NH}$. See [13] for a recent review of neutrino mass models.

${ }^{3}$ See [19] for a recent review of the $\mu-\tau$ symmetry in neutrino physics.
}

C.L. of $1.6 \sigma$. The $\mathrm{NO} \nu \mathrm{A}$ data excludes most of the choices near $\delta=\pi / 2$ for $\mathrm{IH}$ at a C.L. $\geq 3 \sigma$. These results are expected to be further strengthened with as more data becomes available. The recent global analyses of the available neutrino data [24-27] also indicate preference for $\mathrm{NH}$ at more than $3 \sigma$ C.L. and a nonmaximal $\theta_{23}$ around $2 \sigma$ with a slight preference for HO. At the same time, caution needs to be exercised in interpretation of results of $\mathrm{T} 2 \mathrm{~K}$ and $\mathrm{NO} \nu \mathrm{A}$ experiments in resolving mass hierarchy and CPV [28,29]. It is of importance to address the key questions listed above and measure the unknowns in an unambiguous manner.

The upcoming Deep Underground Neutrino Experiment (DUNE) [30-32] has the potential to resolve the key questions mentioned above with a very high precision. DUNE is expected to use the standard low energy (LE) tuned flux (having a peak around 2-3 GeV and sharply falling at energies $E \gtrsim 4 \mathrm{GeV}$ ) with a total runtime of seven years distributed equally between the $\nu$ and $\bar{\nu}$ modes ( 3.5 years +3.5 years). Among the viable additional beams that can be used at DUNE, there is a possibility of deploying a medium energy tune (ME) based on the NuMI focusing system, which offers substantial statistics even at energies $E \gtrsim 4 \mathrm{GeV}$ (albeit at the cost of some loss of statistics around 2-3 GeV). The role of the ME beam at DUNE as been explored in disentangling nonstandard neutrino interactions (NSI) from the standard oscillation [33], constraining parameter degeneracies in the presence of NSI [34] and constraining unitarity using the $\nu_{\mu} \rightarrow \nu_{\tau}$ channel $[35,36]$.

To exploit the full potential of DUNE, we make use of this ME beam with a focus to exploit the high statistics it offers at higher energies. Since the neutrinos and antineutrinos encounter different potential due to earth matter effects, the variation of runtime of a long baseline experiment such as DUNE while running in neutrino $(\nu)$ mode versus antineutrino $(\bar{\nu})$ mode could lead to a difference in sensitivities to mass hierarchy (MH), CPV, and octant of $\theta_{23}$. In the present work, we combine LE and ME beam tunes and vary runtime in the $\nu$ and $\bar{\nu}$ modes corresponding to each of the beams with the goal to improve the sensitivities of DUNE to $\mathrm{MH}, \mathrm{CPV}$, and octant of $\theta_{23}$.

Let us summarize some previous studies which focus on the variation of runtime between the $\nu$ and $\bar{\nu}$ modes. In [37-41], the authors have carried out optimization of runtime combinations in $\nu$ and $\bar{\nu}$ mode for the currently running long baseline experiments, $\mathrm{NO} \nu \mathrm{A}$ and $\mathrm{T} 2 \mathrm{~K}$. They demonstrate that the degeneracies are better resolved with particular choice of runtime combinations. In the context of DUNE, the authors of $[42,43]$ demonstrate that improved sensitivities to $\mathrm{CPV}, \mathrm{MH}$, and octant of $\theta_{23}$ can potentially be reached for particular combinations of runtime in $\nu$ and $\bar{\nu}$ mode. [42] further discusses the possibility of a combined analysis with $\mathrm{T} 2 \mathrm{~K}$ and $\mathrm{NO} \nu \mathrm{A}$, while [43] illustrates the 
importance of $\bar{\nu}$ runtimes at DUNE. [44] discusses about different runtime combinations that can give better precision in measuring the $C P$-violating phase and the octant of $\theta_{23}$ at the erstwhile LBNE [45] (which was the predecessor of DUNE). [46] gives a detailed account of various possible optimized configurations of DUNE, including an analysis of total cumulative runtime. A study has been carried out with the variation of runtime using only the LE flux in the $\nu$ and $\bar{\nu}$ modes [47].

The present work goes beyond studies existing in the literature in two aspects. First, we use an additional beam tune (ME beam) in conjunction with the standard LE beam (thus utilizing the wide band nature of DUNE to a greater extent) and analyze the variation of runtime for both the fluxes in the $\nu$ and $\bar{\nu}$ modes in order to improve the sensitivities. Second, since the ME beam we implement in our simulation has been optimized to detect a large number of $\nu_{\tau}$ events, we include a $\nu_{\mu} \rightarrow \nu_{\tau}$ (as well as $\bar{\nu}_{\mu} \rightarrow \bar{\nu}_{\tau}$ ) appearance channel, in addition to $\nu_{\mu} \rightarrow \nu_{e}\left(\bar{\nu}_{\mu} \rightarrow \bar{\nu}_{e}\right)$ and $\nu_{\mu} \rightarrow \nu_{\mu}\left(\bar{\nu}_{\mu} \rightarrow \bar{\nu}_{\mu}\right)$ channels, in our analysis to estimate the sensitivities to $\mathrm{CPV}, \mathrm{MH}$, and octant of $\theta_{23}$.

This article is organized as follows. In Sec. II, we give briefly describe effective Hamiltonian and the parameters governing standard neutrino oscillations. Section III discusses beam tunes, i.e., the LE and ME fluxes we have used in our analysis. Our analysis methodology is discussed in Sec. IV. In Sec. V, we show our main results, the optimized runtime combinations that generate the best sensitivities to $\mathrm{CPV}, \mathrm{MH}$, and octant of $\theta_{23}$. Finally, we summarize our results and conclude. In the Appendix, we discuss the role of the three oscillation channels to resolve the questions of $\mathrm{CPV}, \mathrm{MH}$, and octant of $\theta_{23}$ in the level of probabilities.

\section{EFFECTIVE HAMILTONIAN IN THE STANDARD OSCILLATION FRAMEWORK}

The effective Hamiltonian describing neutrino propagation in the flavor basis is expressed as

$$
\begin{aligned}
\mathcal{H}^{\mathrm{f}} & =\mathcal{H}^{\mathrm{v}}+\mathcal{H}^{\mathrm{SI}}, \\
& =\frac{\Delta m_{31}^{2}}{2 E}\left\{\mathcal{U}\left(\begin{array}{lll}
0 & & \\
& \alpha & \\
& & 1
\end{array}\right) \mathcal{U}^{\dagger}+A\left(\begin{array}{lll}
1 & 0 & 0 \\
0 & 0 & 0 \\
0 & 0 & 0
\end{array}\right)\right\},
\end{aligned}
$$

where $\alpha=\Delta m_{21}^{2} / \Delta m_{31}^{2}$ and $A=2 \sqrt{2} E G_{F} n_{e} / \Delta m_{31}^{2}$. $2 \sqrt{2} E G_{F} n_{e}$ is the standard charged current (CC) potential due to the coherent forward scattering of neutrinos propagating through a medium of electron density $n_{e}, G_{F}$ being the Fermi constant.

$\mathcal{U}$ is the three flavor neutrino mixing matrix and is responsible for diagonalizing the vacuum part $\left(\mathcal{H}_{\mathrm{v}}\right)$ of the Hamiltonian. It is parametrized by three angles $\theta_{12}, \theta_{23}, \theta_{13}$ and one phase $\delta .{ }^{4}$ If neutrinos are Majorana particles, there can be two additional Majorana-type phases in the three flavor case, but they are of no consequence in neutrino oscillations. In the commonly used Pontecorvo-MakiNakagawa-Sakata (PMNS) parametrization [7], $\mathcal{U}$ is given by

$$
\begin{aligned}
\mathcal{U}= & \left(\begin{array}{ccc}
1 & 0 & 0 \\
0 & c_{23} & s_{23} \\
0 & -s_{23} & c_{23}
\end{array}\right)\left(\begin{array}{ccc}
c_{13} & 0 & s_{13} e^{-i \delta} \\
0 & 1 & 0 \\
-s_{13} e^{i \delta} & 0 & c_{13}
\end{array}\right) \\
& \times\left(\begin{array}{ccc}
c_{12} & s_{12} & 0 \\
-s_{12} & c_{12} & 0 \\
0 & 0 & 1
\end{array}\right),
\end{aligned}
$$

where $s_{i j}=\sin \theta_{i j}, c_{i j}=\cos \theta_{i j}$.

\section{NEUTRINO BEAM TUNES AND EVENT SPECTRA AT DUNE}

The simulations have been carried out using the widely used General Long Baseline Experiment Simulator (GLoBES) [48,49] which solves the full three flavor neutrino propagation equations numerically using the preliminary reference earth model [50] density profile of the Earth. ${ }^{5}$ The most recent DUNE configuration files from the DUNE collaboration [55] have been used. A total runtime of seven years with an on-axis 40 kiloton liquid argon far detector (FD) housed at the Homestake Mine in South Dakota over a baseline of $1300 \mathrm{~km}$ has been incorporated in the simulations.

We use two broad-band beam tunes: (i) the standard LE beam tune used in DUNE CDR [55] and (ii) the ME beam tune optimized for $\nu_{\tau}$ appearance. The beams are obtained from a G4LBNF simulation $[56,57]$ of the LBNF beam line using NuMI-style focusing. These two broad-band beam tunes are consistent with what could be achieved by the LBNF facility and we illustrate their comparison in Fig. 1. We have chosen a design for the ME beam that is nominally compatible with the space and infrastructure capabilities of the LBNF/DUNE beam line and that is based on a real working beamline design currently deployed in $\mathrm{NuMI} / \mathrm{NO} \nu \mathrm{A}$. The beam line parameters assumed for the different design fluxes used in our analyses are listed in Table I.

In Fig. 2, we show the $\nu_{\mu} \rightarrow \nu_{e}\left(\right.$ and $\left.\bar{\nu}_{\mu} \rightarrow \bar{\nu}_{e}\right)$ event spectra at DUNE when the beam tune is either the standard

\footnotetext{
${ }^{4}$ In the general case of $n$ flavors the leptonic mixing matrix $\mathcal{U}$ depends on $(n-1)(n-2) / 2$ Dirac-type $C P$-violating phases. If the neutrinos are Majorana particles, there are $(n-1)$ additional, so called Majorana-type $C P$-violating phases.

${ }^{5}$ We use the matter density as given by preliminary reference earth model. In principle, we can allow for uncertainty in the Earth matter density in our calculations but it would not impact our results drastically [51-54].
} 


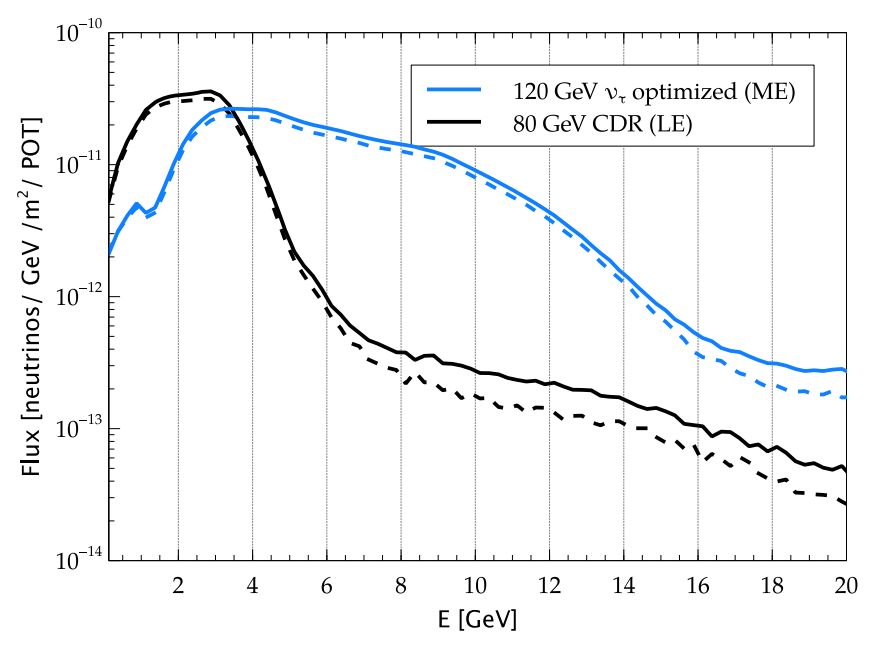

FIG. 1. Comparison of the different beam tunes-the $80 \mathrm{GeV}$ flux used in DUNE conceptual design report (CDR) is referred to as LE flux while $120 \mathrm{GeV}, \nu_{\tau}$ optimized flux is referred to as the ME flux. The solid (dashed) curves represent the $\nu_{\mu}\left(\bar{\nu}_{\mu}\right)$ flux.

LE beam (black) or the $\nu_{\tau}$-optimized ME beam (blue). In order to make a fair comparison, the total runtime has been held fixed to seven years in generating each of these four spectra. We note that though the LE flux gives an excess of events around $E \lesssim 3 \mathrm{GeV}$, the ME beam offers complementarity by generating more events beyond $E \gtrsim 3 \mathrm{GeV}$. In fact, for the chosen values of the parameters, the total number of events summed over the energy bins up to $8 \mathrm{GeV}$ (as indicated in the figure), is slightly more for the ME beam with respect to the LE beam. ${ }^{6}$ This trend is visible for both $\nu$ and $\bar{\nu}$ modes irrespective of the choice of the hierarchy. In view of the above stated observations, we investigate whether a combination of $\mathrm{LE}$ and ME beam could lead to an improvement in the sensitivities to CPV, $\mathrm{MH}$ and octant of $\theta_{23}$ over and above what is expected from the LE beam alone. In Fig. 3, we show the $\nu_{\mu} \rightarrow \nu_{\tau}$ (and $\bar{\nu}_{\mu} \rightarrow \bar{\nu}_{\tau}$ ) event spectra at DUNE when the beam tune is either the standard LE beam (black) or the $\nu_{\tau}$-optimized ME beam (blue). It is worth mentioning that ME beam opens up the possibility to exploit this channel in addition to the $\nu_{\mu} \rightarrow \nu_{e}$ channel which further aids in improving the outcome of the sensitivity studies.

For classification of events using the two beam tunes, we follow Alion et al. [55]. In the GLoBES configurations [55], event classification was listed in distinct categories including electron neutrino appearance signal, $\nu_{\mu} \rightarrow \nu_{e}$ (CC), muon neutrino charged current signal, $\nu_{\mu} \rightarrow \nu_{\mu}$ (CC) as well as neutrino neutral current (NC) background, $\nu_{\mu} / \nu_{e} \rightarrow X$ (NC) both in appearance and disappearance modes. It should be noted that tau neutrino appearance

\footnotetext{
${ }^{6}$ If the parameters are allowed to vary within the $3 \sigma$ allowed range, it is found that this conclusion holds in much of the parameter space.
}

TABLE I. Beam line parameters assumed for the different design fluxes used in our sensitivity calculations $[30,31,55]$. The target is a thin graphite cylinder two interaction lengths long.

\begin{tabular}{lcc}
\hline \hline Parameter & $\begin{array}{c}\text { LE }(\mathrm{CPV} \\
\text { optimized design })\end{array}$ & $\begin{array}{c}\text { ME } \\
\left(\nu_{\tau} \text { optimized }\right)\end{array}$ \\
\hline Proton beam energy & $80 \mathrm{GeV}$ & $120 \mathrm{GeV}$ \\
Proton beam power & $1.07 \mathrm{MW}$ & $1.2 \mathrm{MW}$ \\
Protons on target & $1.47 \times 10^{21}$ & $1.10 \times 10^{21}$ \\
$\quad$ POT $)$ per year & 3 horns & $2 \mathrm{NuMI}$ horns \\
Focusing & genetic optimization & $17 \mathrm{~m} \mathrm{apart}$ \\
& $294 \mathrm{kA}$ & $230 \mathrm{kA}$ \\
Horn current & $194 \mathrm{~m}$ & $200 \mathrm{~m}$ \\
Decay pipe length & $4 \mathrm{~m}$ & $4 \mathrm{~m}$ \\
Decay pipe diameter & & \\
\hline \hline
\end{tabular}

$\nu_{\mu} \rightarrow \nu_{\tau}$ (CC) was included in [55], but as a background. The corresponding systematics/efficiencies were provided as supplementary files in [55]. In the present study, we have incorporated a new category for tau neutrino appearance signal, $\nu_{\mu} \rightarrow \nu_{\tau}(\mathrm{CC})$. The backgrounds corresponding to this category include $\nu_{\mu} \rightarrow \nu_{e}(\mathrm{CC}), \nu_{\mu} \rightarrow \nu_{\mu}$ (CC) (due to the leptonic decay of tau lepton with branching fraction $\sim 35 \%$ ), as well as NC background (due to the hadronic decay of the tau lepton with branching fraction $~ 65 \%$ ). Contamination due to wrong sign leptons is also taken into account as background.

\section{ANALYSIS METHOD}

To estimate the sensitivities of DUNE to MH, CPV, and octant of $\theta_{23}$, we perform the standard $\Delta \chi^{2}$ analysis. Even though all results are produced numerically with the help of GLoBES software, in order to gain insight, let us examine the analytical form of the $\Delta \chi^{2}$.

$$
\begin{aligned}
\Delta \chi^{2}\left(p^{\text {true }}\right)= & \operatorname{Min}_{p^{\text {test }}, \eta}\left[2 \sum _ { y } ^ { \text { flux } } \sum _ { x } ^ { \text { mode channel } } \sum _ { j } ^ { \text { bin } } \left\{\sum _ { i } \left\{N_{i j x y}^{\text {test }}\left(p^{\text {test }} ; \eta\right)\right.\right.\right. \\
& \left.-N_{i j x y}^{\text {true }}\left(p^{\text {true }}\right)+N_{i j x y}^{\text {true }}\left(p^{\text {true }}\right) \ln \frac{N_{i j x y}^{\text {true }}\left(p^{\text {true }}\right)}{N_{i j x y}^{\text {test }}\left(p^{\text {test }} ; \eta\right)}\right\} \\
& \left.+\sum_{l} \frac{\left(p_{l}^{\text {true }}-p_{l}^{\text {test }}\right)^{2}}{\sigma_{p_{l}}^{2}}+\sum_{k} \frac{\eta_{k}^{2}}{\sigma_{k}^{2}}\right]
\end{aligned}
$$

where $N^{\text {true }}$ and $N^{\text {test }}$ are the set of true and test events, respectively. Index $i$ is summed over the energy bins in the range $0-20 \mathrm{GeV}^{8}$ The indices $j$ and $x$ are summed over the channels $\left(\nu_{\mu} \rightarrow \nu_{e}, \nu_{\mu} \rightarrow \nu_{\mu}, \nu_{\mu} \rightarrow \nu_{\tau}\right)$ and the modes ( $\nu$ and

\footnotetext{
${ }^{7}$ This is the Poissonian definition of $\Delta \chi^{2}$, which, in the limit of large sample size, reduces to the Gaussian form.

${ }^{8}$ We have a total of 71 energy bins in the range 0-20 GeV: 64 bins each having a width of $0.125 \mathrm{GeV}$ in the energy range of 0 to $8 \mathrm{GeV}$ and seven bins with variable widths beyond $8 \mathrm{GeV}$ [55].
} 


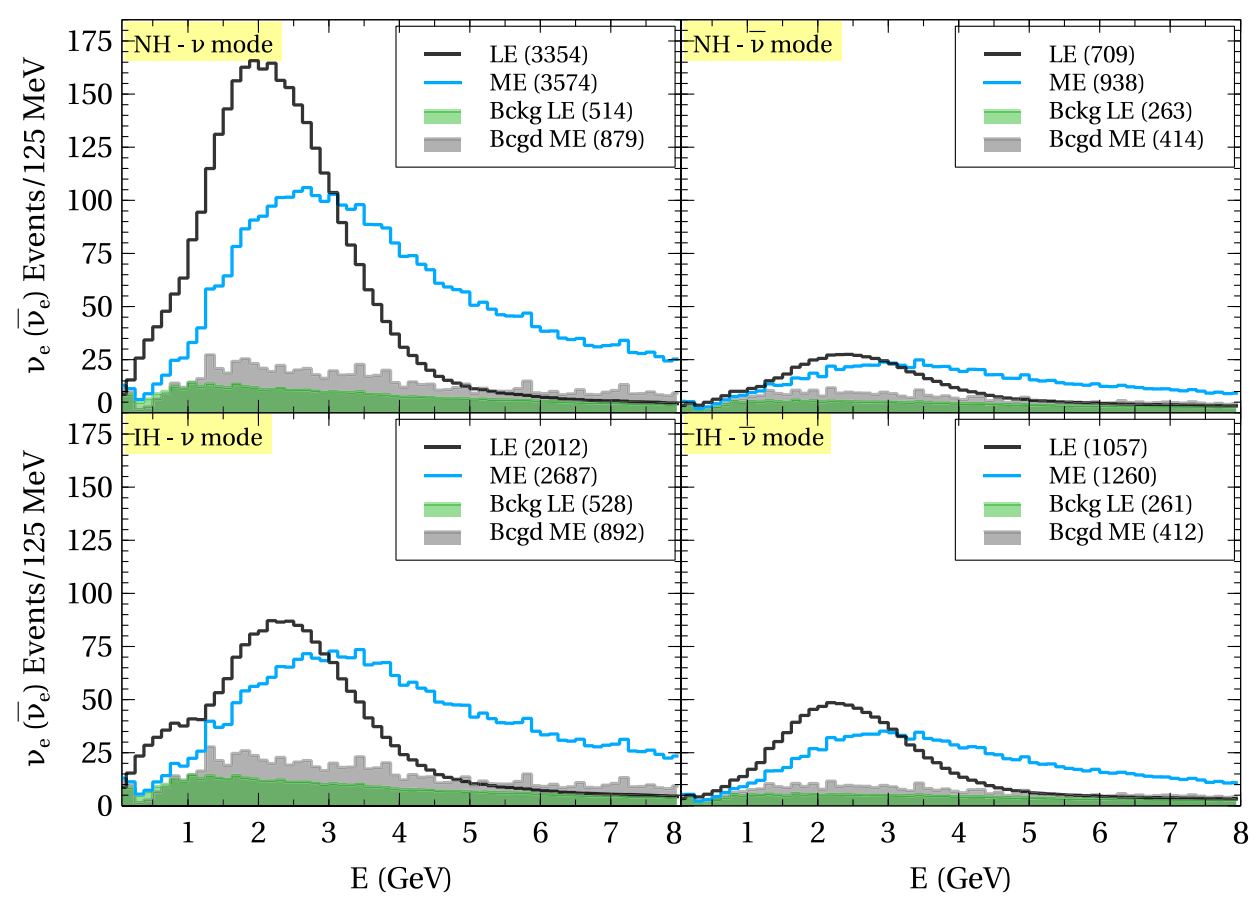

FIG. 2. The $\nu_{\mu} \rightarrow \nu_{e}$ event spectra at DUNE are shown for different cases - the top (bottom) row corresponds to NH (IH) while the left (right) column corresponds to $\nu(\bar{\nu})$ mode. The events obtained using the standard LE beam (the $\nu_{\tau}$-optimized ME beam) are depicted using black (blue) curves in each of the four panels. The total background corresponding with LE (ME) beam are shown as green (gray) shaded regions. In generating each of the event/background spectra, a total runtime of seven years have been used. The numbers in the parentheses in the legends are the corresponding total number of events summed over the energy bins up to $8 \mathrm{GeV}$. The best-fit values of the oscillation parameters have been used to generate these event spectra (see Table II).

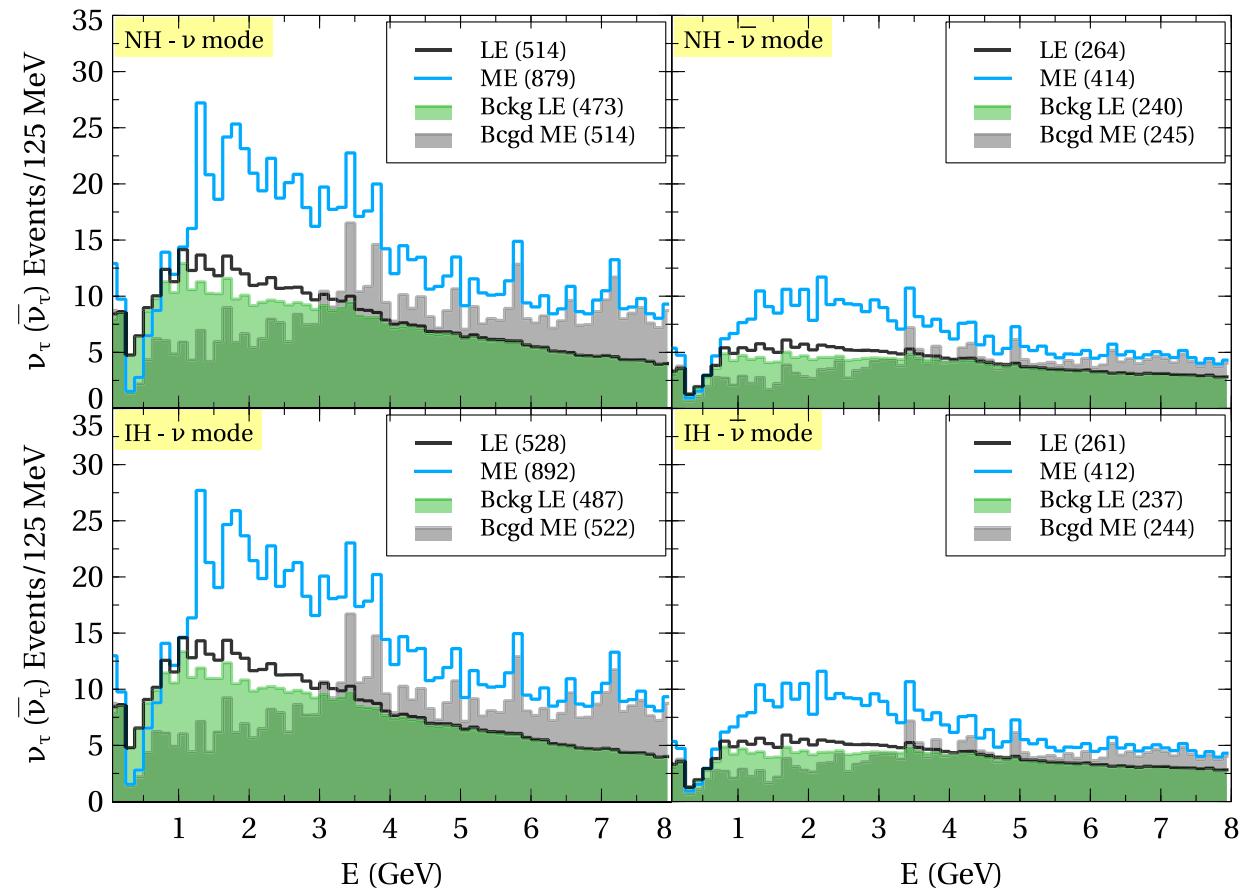

FIG. 3. The $\nu_{\mu} \rightarrow \nu_{\tau}$ event spectra at DUNE are shown for different cases - the top (bottom) row corresponds to NH (IH) while the left (right) column corresponds to $\nu(\bar{\nu})$ mode. The events obtained using the standard LE beam (the $\nu_{\tau}$-optimized ME beam) are depicted using black (blue) curves in each of the four panels. The total background corresponding with LE (ME) beam are shown as green (gray) shaded regions. In generating each of the event/background spectra, a total runtime of seven years have been used. The numbers in the parentheses in the legends are the corresponding total number of events summed over the energy bins up to $8 \mathrm{GeV}$. The best-fit values of the oscillation parameters have been used to generate these event spectra (see Table II). 
TABLE II. Standard oscillation parameters and their uncertainties used in our study. The values were taken from the global fit analysis in [24]. If the $3 \sigma$ upper and lower limit of a parameter is $x_{u}$ and $x_{l}$, respectively, the $1 \sigma$ uncertainty is $\left(x_{u}-x_{l}\right) / 3\left(x_{u}+x_{l}\right) \%$ [31].

\begin{tabular}{lccc}
\hline \hline Parameter & Best-fit value & $3 \sigma$ interval & $1 \sigma$ uncertainty \\
\hline$\theta_{12}$ [Deg.] & 34.3 & $31.4-37.4$ & $2.9 \%$ \\
$\theta_{13}$ (NH) [Deg.] & 8.58 & $8.16-8.94$ & $1.5 \%$ \\
$\theta_{13}$ (IH) [Deg.] & 8.63 & $8.21-8.99$ & $1.5 \%$ \\
$\theta_{23}$ (NH) [Deg.] & 48.8 & $41.63-51.32$ & $3.5 \%$ \\
$\theta_{23}$ (IH) [Deg.] & 48.8 & $41.88-51.30$ & $3.5 \%$ \\
$\Delta m_{21}^{2}\left[\mathrm{eV}^{2}\right]$ & $7.5 \times 10^{-5}$ & {$[6.94-8.14] \times 10^{-5}$} & $2.7 \%$ \\
$\Delta m_{31}^{2}(\mathrm{NH})\left[\mathrm{eV}^{2}\right]$ & $+2.56 \times 10^{-3}$ & {$[2.46-2.65] \times 10^{-3}$} & $1.2 \%$ \\
$\Delta m_{31}^{2}(\mathrm{IH})\left[\mathrm{eV}^{2}\right]$ & $-2.46 \times 10^{-3}$ & $-[2.37-2.55] \times 10^{-3}$ & $1.2 \%$ \\
$\delta(\mathrm{NH})[\mathrm{Rad}]$. & $-0.8 \pi$ & {$[-\pi, 0] \cup[0.8 \pi, \pi]$} & - \\
$\delta(\mathrm{IH})$ [Rad.] & $-0.46 \pi$ & {$[-0.86 \pi,-0.1 \pi]$} & - \\
\hline \hline
\end{tabular}

$\bar{\nu})$, respectively. The sum over the index $y$ takes into account the multiple fluxes (LE and ME tuned beams), whenever multiple fluxes are used. The term inside the curly braces in Eq. (3) is the statistical part of $\Delta \chi^{2}$. The term $\left(N^{\text {test }}-N^{\text {true }}\right)$ takes into account the algebraic difference while the third term inside the curly braces considers the fractional difference between the test and true sets of events. $p^{\text {true }}$ and $p^{\text {test }}$ are the set of true and test oscillation parameters, respectively, and $\sigma_{p_{l}}$ is the uncertainty in the prior measurement of the parameter $p_{l}$. The values of the true or best-fit oscillation parameters and their uncertainties as used in the present analysis are tabulated in Table II. The index $l$ is summed over the number of test oscillation parameters to be marginalized. This is known as the prior term. The index $k$ is summed over the number of systematics/nuisance parameters present. This way of treating the nuisance parameters in the $\Delta \chi^{2}$ calculation is known as the method of pulls [58-61]. Regarding the systematics, the $\nu_{e}$ and $\bar{\nu}_{e}$ signal modes have a normalization uncertainties of $2 \%$ each, whereas the $\nu_{\mu}$ and $\bar{\nu}_{\mu}$ signals have a normalization uncertainty of $5 \%$ each. The $\nu_{\tau}$ and $\bar{\nu}_{\tau}$ signals have a normalization uncertainties of $20 \%$ each. The background normalization uncertainties vary from 5\%-20\% and include correlations among various sources of background (coming from beam $\nu_{e} / \bar{\nu}_{e}$ contamination, flavor misidentification, $\mathrm{NC}$ and $\nu_{\tau}$ ). The final estimate of $\Delta \chi^{2}$ which is thus a function of the true values of the oscillation parameters, is obtained after a minimization (i.e., marginalization over the $3 \sigma$ range of values) over the set of test parameters $\left(p^{\text {test }}\right)$ and the set of systematics $(\eta)$. Technically this $\Delta \chi^{2}$ is the frequentist method of hypotheses testing $[59,62]$.

For calculating the sensitivity to $\mathrm{MH}$, we marginalize the test $\Delta m_{31}^{2}$ in the opposite hierarchy in the $3 \sigma$ range of values. Test parameters $\theta_{23}, \theta_{13}$ are marginalized over their $3 \sigma$ ranges, while the $C P$ phase $\delta$ is marginalized over the full range of $[-\pi, \pi]$. For the sensitivity to CPV, the test $\delta$ is allowed to marginalize over only the $C P$-conserving values of 0 and $\pi$ while the true $\delta$ can take any value in the range $[-\pi, \pi]$. The other test parameters in that case $\left(\Delta m_{31}^{2}\right.$, $\left.\theta_{13}, \theta_{23}\right)$ are marginalized over the $3 \sigma$ range. For the calculation of the sensitivity to $\theta_{23}$ octant, the test $\theta_{23}$ is marginalized in the opposite octant. The marginalization of the test parameters $\Delta m_{31}^{2}$ and $\theta_{13}$ are carried out over their respective $3 \sigma$ range, while that of test $\delta$ is done in the whole allowed range of $[-\pi, \pi]$.

We keep the total runtime at DUNE fixed at seven years and numerically calculate $\Delta \chi^{2}$ as a function of true $\delta \in[-\pi, \pi]$ after varying the distribution of runtime (with a stepsize of 0.5 year) among the following four variables:

(i) Runtime using LE beam and in neutrino mode $\left(\mathcal{R}_{\mathrm{LE}}\right)$.

(ii) Runtime using LE beam and in antineutrino mode $\left(\overline{\mathcal{R}}_{\mathrm{LE}}\right)$.

(iii) Runtime using $\mathrm{ME}$ beam and in neutrino mode $\left(\mathcal{R}_{\mathrm{ME}}\right)$.

(iv) Runtime using $\mathrm{ME}$ beam and in antineutrino mode $\left(\overline{\mathcal{R}}_{\mathrm{ME}}\right)$.

Note that, since $\mathcal{R}_{\mathrm{LE}}+\overline{\mathcal{R}}_{\mathrm{LE}}+\mathcal{R}_{\mathrm{ME}}+\overline{\mathcal{R}}_{\mathrm{ME}}=7$ (years), only three of the above variables are independent. In order to figure out the optimized runtime combination, we define that combination of $\mathcal{R}_{\mathrm{LE}}, \overline{\mathcal{R}}_{\mathrm{LE}}, \mathcal{R}_{\mathrm{ME}}, \overline{\mathcal{R}}_{\mathrm{ME}}$, which gives the largest area under the sensitivity curve in the $\left(\Delta \chi^{2}\right.$-true $\left.\delta\right)$ plane for all three unknowns: CPV, MH, and octant of $\theta_{23}$. We refer to the optimized runtime combination estimated in this manner as $R_{\mathrm{CPV}}^{\text {area }}, R_{\mathrm{MH}}^{\text {area }}$, or $R_{\mathrm{OCT}}^{\text {area }}$, respectively for the three quantities. Additionally, for the CPV case, we define another optimized combination of runtimes (i.e., $\left.\mathcal{R}_{\mathrm{LE}}, \overline{\mathcal{R}}_{\mathrm{LE}}, \mathcal{R}_{\mathrm{ME}}, \overline{\mathcal{R}}_{\mathrm{ME}}\right)$ that resolves $\mathrm{CPV}$ above $3 \sigma$ for the largest fraction of true $\delta$ parameter space. We refer to this optimized combination as $R_{\mathrm{CPV}}^{\text {fraction }}$.

\section{OPTIMIZED RUNTIME COMBINATIONS: SENSITIVITY TO CPV, MH, AND OCTANT OF $\boldsymbol{\theta}_{23}$}

We present our main results by estimating the optimized runtime combinations of $\left(\mathcal{R}_{\mathrm{LE}}, \overline{\mathcal{R}}_{\mathrm{LE}}, \mathcal{R}_{\mathrm{ME}}, \overline{\mathcal{R}}_{\mathrm{ME}}\right)$ that give the best sensitivities to resolve $\mathrm{CPV}, \mathrm{MH}$, and octant of $\theta_{23}$. 


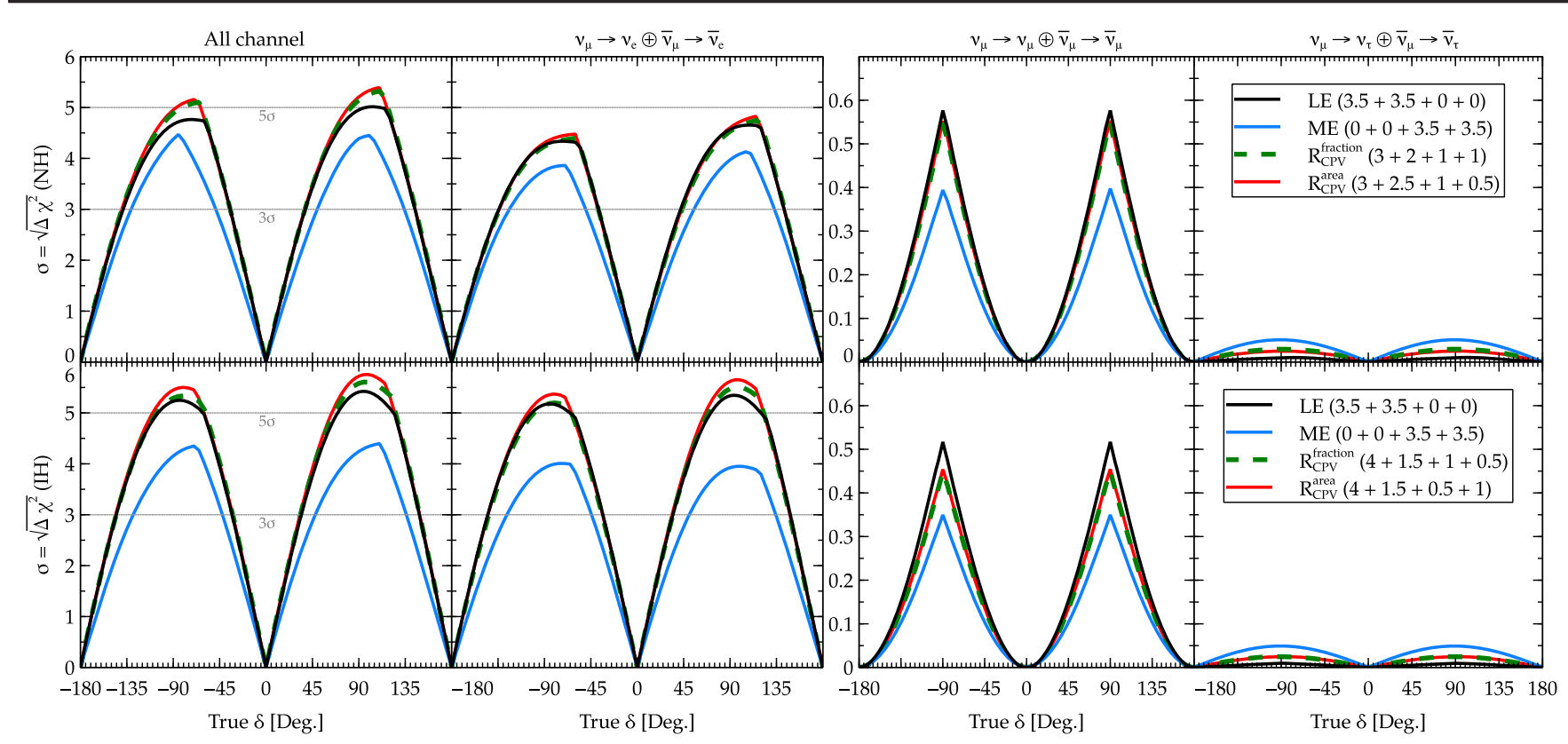

FIG. 4. The sensitivity to CPV at DUNE are shown with the variation of true $\delta$, for optimized runtime combination for LE + MEfound either by maximizing the area under the sensitivity curve (solid red) or by maximizing the fraction of true $\delta$ space for which the sensitivity is above $3 \sigma$ (dashed green). The sensitivities obtained for LE only (black) or ME only (blue) beam with equal runtime in $\nu$ and $\bar{\nu}$ modes are also shown. The legends signify the runtime combination for each case in the form $\left(\mathcal{R}_{\mathrm{LE}}+\overline{\mathcal{R}}_{\mathrm{LE}}+\mathcal{R}_{\mathrm{ME}}+\overline{\mathcal{R}}_{\mathrm{ME}}\right)$. The top (bottom) row depicts the case of true $\mathrm{NH}$ (IH). The first column shows the combined results considering all channels while the second, third, and fourth column shows the contributions from the individual channels $\nu_{\mu} \rightarrow \nu_{e}, \nu_{\mu} \rightarrow \nu_{\mu}$ and $\nu_{\mu} \rightarrow \nu_{\tau}$, respectively.

In what follows, we obtain the optimal combinations of runtimes and which are reported as $\left(\mathcal{R}_{\mathrm{LE}}+\overline{\mathcal{R}}_{\mathrm{LE}}+\mathcal{R}_{\mathrm{ME}}+\right.$ $\overline{\mathcal{R}}_{\mathrm{ME}}$ ) for the three different questions addressed in the present work.

\section{A. Sensitivity to $C P$ violation}

In Fig. 4, we illustrate the optimized runtime combination of $\left(\mathcal{R}_{\mathrm{LE}}+\overline{\mathcal{R}}_{\mathrm{LE}}+\mathcal{R}_{\mathrm{ME}}+\overline{\mathcal{R}}_{\mathrm{ME}}\right)$ that give the best sensitivity to CPV at DUNE by (a) maximizing the area under the sensitivity curve (red solid), and (b) maximizing the fraction of true $\delta$ parameter space that resolves the CPV sensitivity above $3 \sigma$ (green dashed). For comparison, we also show the CPV sensitivities by using only the standard LE beam (black) or the ME beam only (blue) - the distribution of runtime being equally (3.5 years) shared between the $\nu$ and $\bar{\nu}$ modes for each case. The first column shows the main results where (neutrino and antineutrino) contributions from all three oscillation channels $\left(\nu_{\mu} \rightarrow\right.$ $\left.\nu_{e}, \nu_{\mu} \rightarrow \nu_{\mu}, \nu_{\mu} \rightarrow \nu_{\tau}\right)$ are considered. The second, third, and fourth column shows the (neutrino and antineutrino) contribution from the individual channels $\nu_{\mu} \rightarrow \nu_{e}, \nu_{\mu} \rightarrow \nu_{\mu}$ and $\nu_{\mu} \rightarrow \nu_{\tau}$, respectively. The top (bottom) row depicts the case of true $\mathrm{NH}(\mathrm{IH})$.

For $\mathrm{NH}$, the sensitivity to $\mathrm{CPV}$ at the best runtime combination $\left(R_{\mathrm{CPV}}^{\text {area }}\right.$ or $\left.R_{\mathrm{CPV}}^{\text {fraction }}\right)$ is enhanced beyond $5 \sigma$ around the $C P$-violating values $(\approx \pm \pi / 2)$ of true $\delta$. The $5 \sigma$ reach of the sensitivity to $\mathrm{CPV}$ was otherwise not achievable using only the standard LE beam or the ME beam with a runtime of (3.5 years $\nu$ mode +3.5 years $\bar{\nu}$ mode for either of the beams). For the $\mathrm{NH}$ scenario, an optimized runtime combination $\left(R_{\mathrm{CPV}}^{\text {area }} \equiv \mathbf{3}+\mathbf{2 . 5}+\mathbf{1}+\mathbf{0 . 5}\right)$ estimated by maximizing the area of implies that DUNE needs to run for a total of (a) 5.5 years using the LE beam (with 3 years in $\nu$ mode and 2.5 years in $\bar{\nu}$ mode), and (b) 1.5 years using the ME beam (with 1 year in $\nu$ mode and 0.5 year in $\bar{\nu}$ mode). It should be noted that the optimized runtime combination obtained by maximizing the fraction is similar to that obtained while maximizing the area.

For $\mathrm{IH}$, it is found that the $C P$ violation sensitivity does not improve much when we use a combination of beam tunes $(\mathrm{LE}+\mathrm{ME})$. This is because the sensitivity generated by LE beam is somewhat higher for IH than for $\mathrm{NH}$ which gives less scope of improvement.

It is worth mentioning that most of the contribution to the $C P$ violation sensitivity arises from the $\nu_{\mu} \rightarrow \nu_{e}$ appearance channel. In the Appendix, we try to explain why this is the dominant channel while the other two channels (i.e., $\nu_{\mu} \rightarrow \nu_{\mu}$ and $\nu_{\mu} \rightarrow \nu_{\tau}$ ) give rise to small contribution (see also $[63,64])$.

\section{B. Sensitivity to $\mathbf{M H}$}

In Fig. 5, we illustrate the optimized runtime combination (red curves) of $\left(\mathcal{R}_{\mathrm{LE}}+\overline{\mathcal{R}}_{\mathrm{LE}}+\mathcal{R}_{\mathrm{ME}}+\overline{\mathcal{R}}_{\mathrm{ME}}\right)$ that gives 


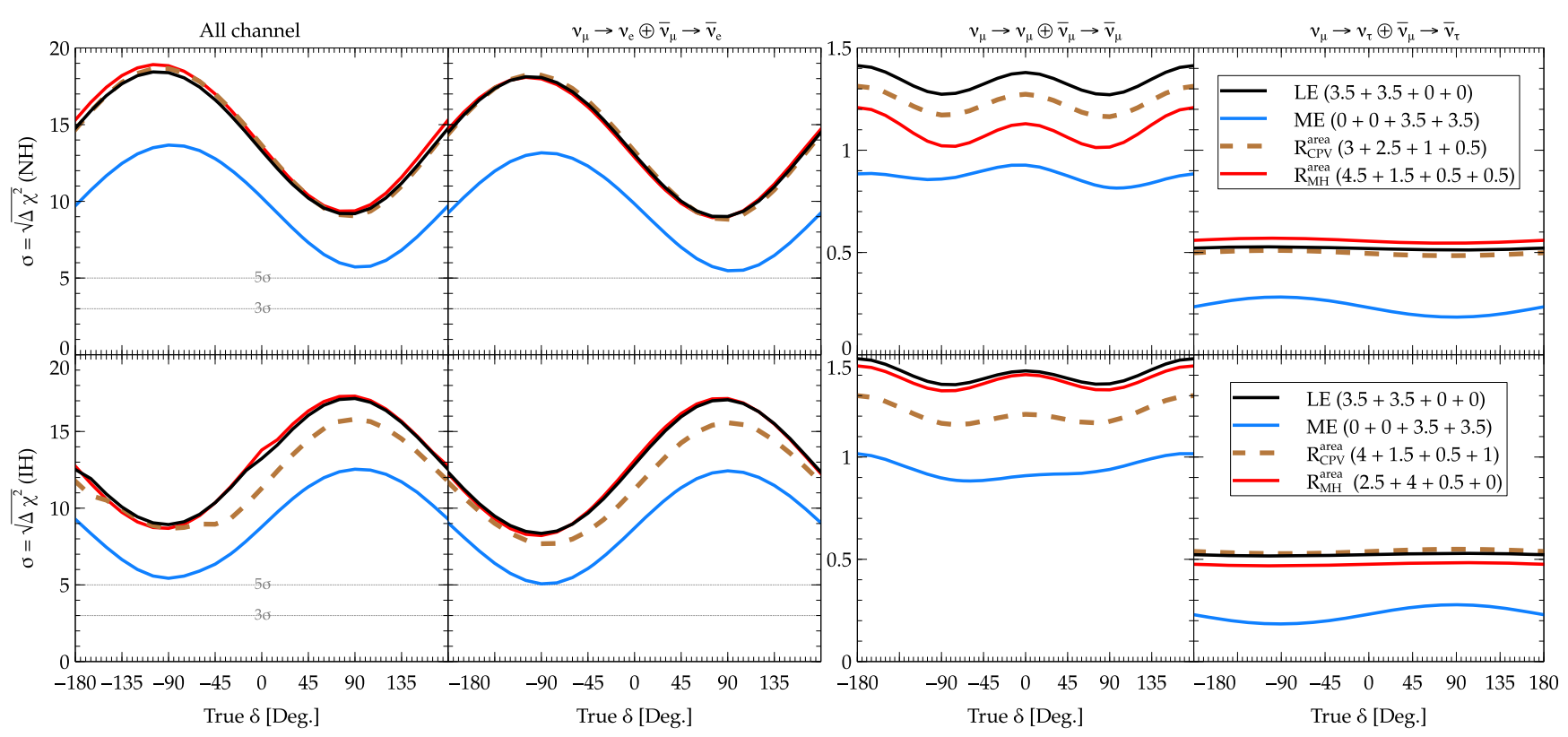

FIG. 5. The sensitivities to MH at DUNE are shown with the variation of true $\delta$, for optimized runtime combination for LE + MEfound by maximizing the area under the sensitivity curve (solid red). The sensitivities obtained for LE only (black) or ME only (blue) beam are also shown with equal runtime in $\nu$ and $\bar{\nu}$ modes. The legends signify the runtime combination for each case in the form $\left(\mathcal{R}_{\mathrm{LE}}+\overline{\mathcal{R}}_{\mathrm{LE}}+\mathcal{R}_{\mathrm{ME}}+\overline{\mathcal{R}}_{\mathrm{ME}}\right)$. For comparison, the $\mathrm{MH}$ sensitivities corresponding to $R_{\mathrm{CPV}}^{\text {area }}$ are also shown (dashed brown). The legends signify the runtime combination for each case in the form $\left(\mathcal{R}_{\mathrm{LE}}+\overline{\mathcal{R}}_{\mathrm{LE}}+\mathcal{R}_{\mathrm{ME}}+\overline{\mathcal{R}}_{\mathrm{ME}}\right)$. The top (bottom) row depicts the case of true $\mathrm{NH}(\mathrm{IH})$. The first column shows the combined results considering all channels while the second, third, and fourth column shows the contributions from the individual channels $\nu_{\mu} \rightarrow \nu_{e}, \nu_{\mu} \rightarrow \nu_{\mu}$, and $\nu_{\mu} \rightarrow \nu_{\tau}$, respectively.
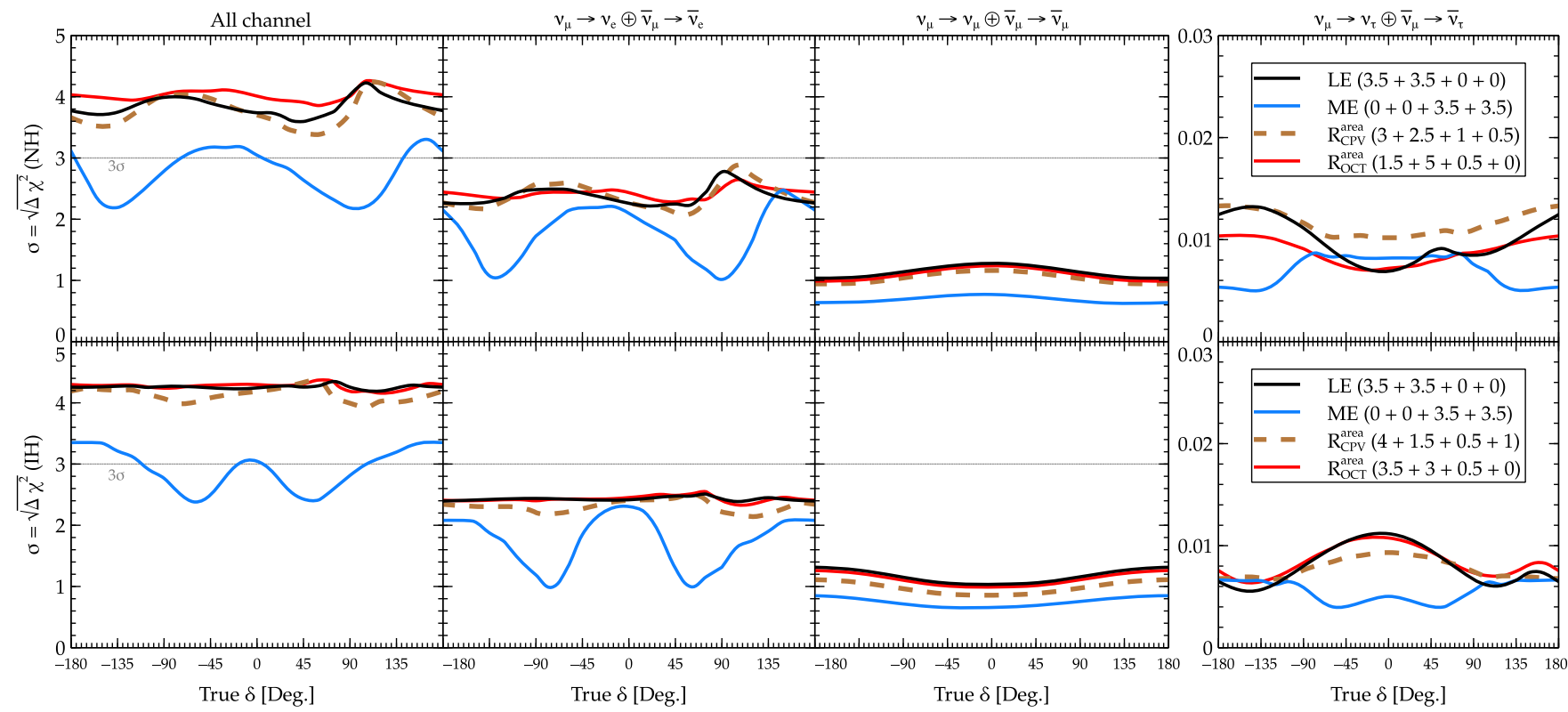

FIG. 6. The sensitivities to $\theta_{23}$-octant degeneracy (true octant being HO) at DUNE are shown with the variation of true $\delta$, for optimized runtime combination for LE $+\mathrm{ME}$ - found by maximizing the area under the sensitivity curve (solid red). The sensitivities obtained for LE only (black) or ME only (blue) beam are also shown with equal runtime in $\nu$ and $\bar{\nu}$ modes. The legends signify the runtime combination for each case in the form $\left(\mathcal{R}_{\mathrm{LE}}+\overline{\mathcal{R}}_{\mathrm{LE}}+\mathcal{R}_{\mathrm{ME}}+\overline{\mathcal{R}}_{\mathrm{ME}}\right)$. For comparison, the octant sensitivities corresponding to $R_{\mathrm{CPV}}^{\text {area }}$ are also shown (dashed brown). The legends signify the runtime combination for each case in the form $\left(\mathcal{R}_{\mathrm{LE}}+\overline{\mathcal{R}}_{\mathrm{LE}}+\mathcal{R}_{\mathrm{ME}}+\overline{\mathcal{R}}_{\mathrm{ME}}\right)$. The top (bottom) row depicts the case of true $\mathrm{NH}(\mathrm{IH})$. The first column shows the combined results considering all channels while the second, third, and fourth column shows the contributions from the individual channels $\nu_{\mu} \rightarrow \nu_{e}, \nu_{\mu} \rightarrow \nu_{\mu}$, and $\nu_{\mu} \rightarrow \nu_{\tau}$, respectively. 
TABLE III. Optimized runtime combinations for sensitivity to CPV, MH, and octant of $\theta_{23}$ at DUNE.

\begin{tabular}{|c|c|c|c|}
\hline Sensitivity to & Optimization combination & $\begin{array}{c}\mathrm{NH} \\
\left(\mathcal{R}_{\mathrm{LE}}+\overline{\mathcal{R}}_{\mathrm{LE}}+\mathcal{R}_{\mathrm{ME}}+\overline{\mathcal{R}}_{\mathrm{ME}}\right) \\
\text { (in years) }\end{array}$ & 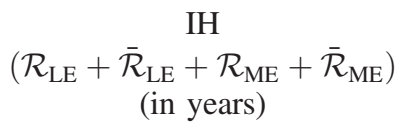 \\
\hline \multirow{2}{*}{ CPV } & $R_{\mathrm{CPV}}^{\text {area }}$ & $3+2.5+1+0.5$ & $4+1.5+0.5+1$ \\
\hline & $R_{\mathrm{CPV}}^{\text {fraction }}$ & $3+2+1+1$ & $4+1.5+1+0.5$ \\
\hline MH & $R_{\mathrm{MH}}^{\text {area }}$ & $4.5+1.5+0.5+0.5$ & $2.5+4+0.5+0$ \\
\hline Octant of $\theta_{23}$ & $R_{\mathrm{OCT}}^{\text {area }}(\mathrm{HO})$ & $1.5+5+0.5+0$ & $3.5+3+0.5+0$ \\
\hline
\end{tabular}

the best MH sensitivity at DUNE. For the sake of comparison, we additionally show the MH sensitivities (dashed brown) corresponding to the best runtime combination $\left(R_{\mathrm{CPV}}^{\text {area }}\right)$ estimated for CPV sensitivity. We also show the sensitivities to MH by using only the standard LE beam (black) or the ME beam (blue), the distribution of runtime being equally ( 3.5 years) shared between the $\nu$ and $\bar{\nu}$ modes. The MH sensitivity of DUNE using only the standard LE beam is already very high for the entire range of values of the $C P$ phase and $R_{\mathrm{MH}}^{\text {area }}$ combination does not offer much improvement. Since the contribution to $\mathrm{MH}$ sensitivity mainly comes from around the first oscillation maximum (2-2.5 GeV) for which the LE beam offers best statistics, the use of ME beam is not expected to lead to much improvement. The best $\mathrm{MH}$ runtime combination is $(\mathbf{4 . 5}+\mathbf{1 . 5}+$ $\mathbf{0 . 5}+\mathbf{0 . 5})$ for $\mathrm{NH}$ and $(2.5+4+0.5+0)$ for $\mathrm{IH}$. For this particular question also, the $\nu_{\mu} \rightarrow \nu_{\mu}$ and $\nu_{\mu} \rightarrow \nu_{\tau}$ channels do not contribute significantly (see the Appendix and [65] to get an insight into the typical shape of the MH sensitivities at long baseline experiments as well as for an understanding of the role of different oscillation channels). As can be seen from Fig. 5, we have also estimated the MH sensitivity corresponding to $R_{\mathrm{CPV}}^{\text {area }}$ (brown dashed curve). It is seen that for $\mathrm{NH}$, it is almost the same as that of $R_{\mathrm{MH}}^{\text {area }}$ while for $\mathrm{IH}$ it is lower than the standard sensitivity (black curve). Interestingly, we observe that, for $R_{\mathrm{MH}}^{\text {area }}, \mathcal{R}_{\mathrm{LE}}$ plays a dominant role when the hierarchy is normal while $\overline{\mathcal{R}}_{\mathrm{LE}}$ plays a dominant role for the $\mathrm{IH}$ case.

\section{Sensitivity to octant of $\boldsymbol{\theta}_{\mathbf{2 3}}$}

We next compute the optimized runtime combinations of $\left(\mathcal{R}_{\mathrm{LE}}+\overline{\mathcal{R}}_{\mathrm{LE}}+\mathcal{R}_{\mathrm{ME}}+\overline{\mathcal{R}}_{\mathrm{ME}}\right)$ that gives the best sensitivity to $\theta_{23}$ octant at DUNE when the true octant is HO $\left(\theta_{23}=48.8^{\circ}\right)$. In Fig. 6, the top (bottom) row depict the case of $\mathrm{NH}(\mathrm{IH})$, while the second, third and fourth columns show the roles of individual oscillation channels. We observe in Fig. 6 that the best runtime combination of $(\mathbf{1 . 5}+\mathbf{5}+\mathbf{0 . 5}+\mathbf{0})$ for $\mathrm{NH}$ scenario underscores the importance of $\bar{\nu}$ runtimes $^{9}$ and this optimized combination

\footnotetext{
${ }^{9}$ As has been discussed in detail in [42], antineutrino run is important in estimating the $\theta_{23}$ octant degeneracy due to complementary nature of the degeneracy for $\nu_{\mu} \rightarrow \nu_{e}$ and $\bar{\nu}_{\mu} \rightarrow$ $\bar{\nu}_{e}$ channels.
}

improves this sensitivity significantly. This is apparent for IH scenario $(2.5+3+1.5+0)$ as well, albeit in a less prominent manner. We also note that unlike the case of $\mathrm{CPV}$ and $\mathrm{MH}$, the $\nu_{\mu} \rightarrow \nu_{\mu}\left(\bar{\nu}_{\mu} \rightarrow \bar{\nu}_{\mu}\right)$ disappearance channel contributes substantially to the octant sensitivities. The $\nu_{\mu} \rightarrow \nu_{\tau}\left(\bar{\nu}_{\mu} \rightarrow \bar{\nu}_{\tau}\right)$ channel, on the other hand, practically has no role (see the Appendix). We also show the octant sensitivities by using only the standard LE beam (black) or the ME beam only (blue), the distribution of runtime being equally shared among the $\nu$ and $\bar{\nu}$ modes. Additionally, for the sake of comparison, the dashed brown curves illustrate the octant sensitivity obtained corresponding to $R_{\mathrm{CPV}}^{\text {area }}$.

Table III summarizes the results for estimated optimized combinations with respect to CPV sensitivity, MH sensitivity, and sensitivity to the octant of $\theta_{23}$, respectively.

\section{SENSITIVITY TO THE CHOICE OF OPTIMAL COMBINATION}

Having completed the task of estimating the optimal combination of runtime that yields the best sensitivity to the three unknowns, we would now like to pose the following question. How sensitive are we to the choice of optimal combination or what direction can we take (in our choice of runtime combinations) in case we have difficulty in implementation of the particular runtime combination. We address these questions in the present section.

We examine the different runtime combinations and analyze how the results improve (i.e., how the area under the $\Delta \chi^{2}$ curve as a function of true $\delta$ for CPV, MH, octant increases) for different runtime combinations. In Fig. 7, we show the heatmap of the area (normalized) under the sensitivity curves (in the $\Delta \chi^{2}$-true $\delta$ plane) for all the runtime combinations $\left(\mathcal{R}_{\mathrm{LE}}+\overline{\mathcal{R}}_{\mathrm{LE}}+\mathcal{R}_{\mathrm{ME}}+\overline{\mathcal{R}}_{\mathrm{ME}}\right)$ considered. The three columns show the case of CPV, MH, and octant sensitivities, while the top (bottom) row depicts the $\mathrm{NH}$ (IH) scenario. The lower triangular portion (red) shows the effect of the $\mathcal{R}_{\mathrm{LE}}$ and $\overline{\mathcal{R}}_{\mathrm{LE}}$ components of the runtime combination along the bottom horizontal axis and the left vertical axis respectively. The upper triangular portion (blue) shows the effect of the $\mathcal{R}_{\mathrm{ME}}$ and $\overline{\mathcal{R}}_{\mathrm{ME}}$ components of the runtime combination along the top horizontal axis and the right vertical axis respectively. The lighter (darker) shades of the colors imply better (worse) sensitivity. Thus, 


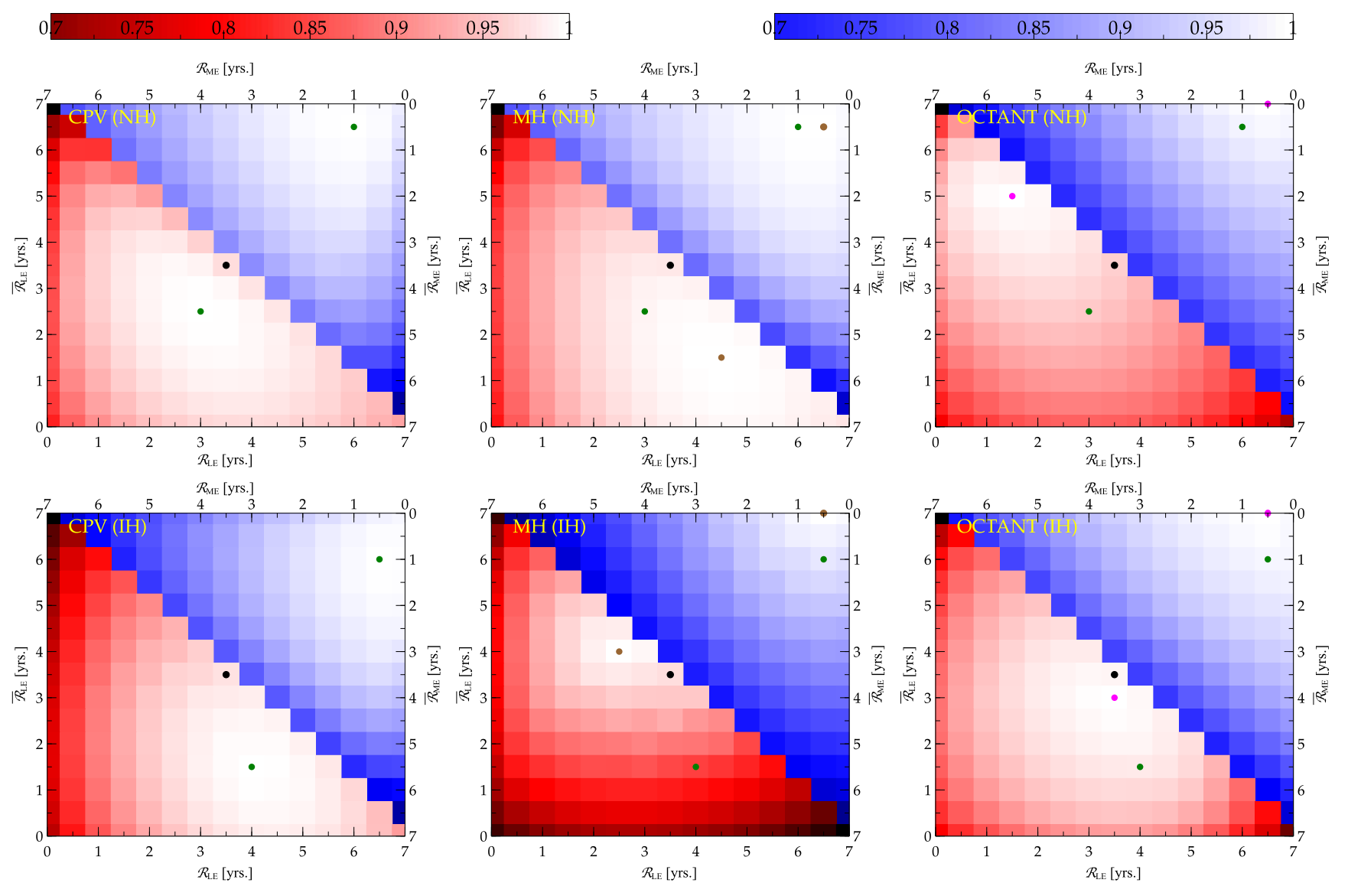

FIG. 7. Heatmap of the normalized area under the sensitivity curves (in the $\left[\Delta \chi^{2}\right.$-true $\delta$ ] plane) for all the runtime combinations considered in the context of $\mathrm{CPV}, \mathrm{MH}$, and $\theta_{23}$ octant sensitivities in the three columns, respectively. The top (bottom) row depicts the case of true $\mathrm{NH}(\mathrm{IH})$. The four components of the runtime combination are shown along the four sides of each panel. The lighter (darker) shades of the colors imply better (worse) sensitivity. We mark the case of standard runtime $\left(\mathcal{R}_{\mathrm{LE}}+\overline{\mathcal{R}}_{\mathrm{LE}}+\mathcal{R}_{\mathrm{ME}}+\overline{\mathcal{R}}_{\mathrm{ME}}=\right.$ $3.5+3.5+0+0)$ with a black dot. The optimized runtime combinations summarized in Table III $\left(R_{\mathrm{CPV}}^{\text {area }}, R_{\mathrm{MH}}^{\text {area }}, R_{\mathrm{OCT}}^{\text {area }}\right)$ giving the best sensitivities are marked with green, brown, and magenta dots in the three columns respectively. The combination $R_{\mathrm{CPV}}^{\text {area }}$ (green dot) is also marked for the case of $\mathrm{MH}$ and octant sensitivities (i.e., second and third column) for comparison.

any runtime combination read off Fig. 7 consists of two parts: $\left(\mathcal{R}_{\mathrm{LE}}+\overline{\mathcal{R}}_{\mathrm{LE}}\right)$ in the red lower triangular region and the corresponding $\left(\mathcal{R}_{\mathrm{ME}}+\overline{\mathcal{R}}_{\mathrm{ME}}\right)$ in the blue upper triangular region such that $\mathcal{R}_{\mathrm{LE}}+\overline{\mathcal{R}}_{\mathrm{LE}}+\mathcal{R}_{\mathrm{ME}}+\overline{\mathcal{R}}_{\mathrm{ME}}=7$. The black dot indicates the case of standard runtime using only LE beam $(\mathbf{3 . 5}+\mathbf{3 . 5}+\mathbf{0}+\mathbf{0})$. The optimized runtime combinations $\left(R_{\mathrm{CPV}}^{\text {area }}, R_{\mathrm{MH}}^{\text {area }}, R_{\mathrm{OCT}}^{\text {area }}\right)$ giving the best sensitivities are marked with a pair of green, brown, and magenta dots in the three columns, respectively. For comparison, the combination $R_{\mathrm{CPV}}^{\text {area }}$ (green dot) is also marked for the case of $\mathrm{MH}$ and octant sensitivities (i.e., second and third column). The fact that the lighter shaded regions are located away from the combination represented by the black dot clearly indicates how the result improves when one combines the ME beam (i.e., nonzero $\mathcal{R}_{\mathrm{ME}}$ and $\overline{\mathcal{R}}_{\mathrm{ME}}$ ) with the LE beam. For $\mathrm{MH}$ (second column), interestingly more $\mathcal{R}_{\mathrm{LE}}$ facilitates the improvement of the sensitivities for true $\mathrm{NH}$ case, while $\overline{\mathcal{R}}_{\mathrm{LE}}$ is slightly favored for true IH. For octant sensitivity (third column of Fig. 7) it is clear that for
$\mathrm{NH}, R_{\mathrm{OCT}}^{\text {area }}$ is dominated by $\overline{\mathcal{R}}_{\mathrm{LE}}$, while for IH, both $\mathcal{R}_{\mathrm{LE}}$ and $\overline{\mathcal{R}}_{\text {LE }}$ play equally important roles. Figure 7 also helps to highlight the need for $\bar{\nu}$ mode runs in probing the $\theta_{23}$ octant sensitivity.

\section{SUMMARY}

$C P$ violation, $\mathrm{MH}$, and octant of $\theta_{23}$ are the crucial unknowns and current and future long baseline experiments such as DUNE are planned to address these questions. In the basic configuration, it is assumed that DUNE would have a runtime of seven years (distributed equally in the $\nu$ and $\bar{\nu}$ mode) with the standard LE beam. The LE beam that is often used in DUNE simulations has a peak around $2-3 \mathrm{GeV}$ (the first oscillation maximum for $\nu_{\mu} \rightarrow \nu_{e}$ transition probability) but very sharply falls off at $E \gtrsim 4 \mathrm{GeV}$. Consequently, the number of events beyond $4 \mathrm{GeV}$ rapidly becomes smaller, providing very little statistics. In the present work, we propose to use a higher energy, ME beam that has a 
substantial flux even beyond $4 \mathrm{GeV}$ in addition to the $\mathrm{LE}$ beam and ask whether this can offer any improvement to the standard sensitivity reach of DUNE in answering question pertaining to $\mathrm{CPV}, \mathrm{MH}$, and octant of $\theta_{23}$.

Keeping the total runtime fixed to seven years, we have distributed the total runtime among the $\nu$ and $\bar{\nu}$ modes with the possibility of utilizing the different beam tunes, LE and ME. We have chosen a design for the ME beam that is nominally compatible with the space and infrastructure capabilities of the LBNF/DUNE beam line and that is based on a real working beam line design currently deployed in $\mathrm{NuMI} / \mathrm{NO} \nu \mathrm{A}$. In each of these sensitivity analyses, we have considered the (neutrino and antineutrino) contributions of all three oscillation channels $\nu_{\mu} \rightarrow \nu_{e}, \nu_{\mu} \rightarrow \nu_{\mu}$, and $\nu_{\mu} \rightarrow \nu_{\tau}$ and shown the contribution of the individual channels to the overall sensitivity. We specify the different runtimes using different beam tunes and modes as $\mathcal{R}_{\mathrm{LE}}, \overline{\mathcal{R}}_{\mathrm{LE}}, \mathcal{R}_{\mathrm{ME}}, \overline{\mathcal{R}}_{\mathrm{ME}}$. The optimized combinations $\mathcal{R}_{\mathrm{LE}}+$ $\overline{\mathcal{R}}_{\mathrm{LE}}+\mathcal{R}_{\mathrm{ME}}+\overline{\mathcal{R}}_{\mathrm{ME}}$ that give the best sensitivities to CPV, $\mathrm{MH}$, and octant of $\theta_{23}$ are then evaluated.

Our results are reported in Sec. V. In Fig. 4, we found that a runtime combination of $(\mathbf{3}+\mathbf{2 . 5}+\mathbf{1}+\mathbf{0 . 5})$ gives the best sensitivity to $C P$ violation if the hierarchy is normal. Also, the sensitivity can reach beyond $5 \sigma$, which was otherwise not possible with the standard DUNE configuration with LE beam alone near $\delta \simeq \pm \pi / 2$ (maximal CPV). In addition to resolving CPV, this particular optimized runtime combination also offers high sensitivity to resolve the MH (see Fig. 5) and octant of $\theta_{23}$ (see Fig. 6). For MH and octant of $\theta_{23}$, the optimized runtime combinations providing the best sensitivities are found to be $(\mathbf{4 . 5}+\mathbf{1 . 5}+$ $\mathbf{0 . 5}+\mathbf{0 . 5})$ and $(\mathbf{1 . 5}+\mathbf{5}+\mathbf{0 . 5}+\mathbf{0})$, respectively (assuming the hierarchy is normal). Finally, Table III summarizes the results for estimated optimized combinations with respect to CPV sensitivity, MH sensitivity, and senstivity to the octant of $\theta_{23}$, respectively.

This study, therefore, underscores the availability of the room for improvement within the DUNE experimental configuration by using a combination of runtime in the $\nu$ and $\bar{\nu}$ mode, exploiting two different (LE and ME) beam tunes. This suggested runtime configuration with the two available beam tunes will eventually help DUNE to answer, with more robustness, its main goals pertaining to leptonic $C P$ violation, determination of $\mathrm{MH}$ and octant of $\theta_{23}$.

We would like to mention that our phenomenological study is concerned primarily with bringing out the physics capabilities introduced by the ME beam tune. Consideration of the cost as well as other detailed technical changes needed to replace the current beam line design with the ME tune proposed above is outside the purview of this paper. It may be worth mentioning that LBNF/DUNE full $C P$ violation sensitivity assumes an upgrade to a $2.4 \mathrm{MW}$ beam, which will require a complete redesign of the targeting and focusing system and thus the facility is designed with the ability to accommodate different targetry and focusing designs.

\section{ACKNOWLEDGMENTS}

M. M. acknowledges the financial support from the Indian National Science Academy (INSA) Young Scientist Project [INSA/SP/YS/2019/269]. The authors acknowledge HPC Cluster Computing System at HRI. This material is based upon work supported by the U.S. Department of Energy, Office of Science, Office of High Energy Physics under Contract No. DE-SC0012704; the Indian funding from University Grants Commission under the second phase of University with Potential of Excellence (UPE II) at JNU and Department of Science and Technology under DST-PURSE at JNU. This work has received partial funding from the European Union's Horizon 2020 research and innovation programme under the Marie Skodowska-Curie Grant Agreements No. 690575 and No. 674896. P. M. acknowledges the kind hospitality from the particle physics group at Brookhaven National Laboratory during the initial stages of this work.

\section{APPENDIX: ROLE OF DIFFERENT CHANNELS IN PROBABILITY}

Here we discuss how the individual channels $\left(\nu_{\mu} \rightarrow\right.$ $\nu_{e}, \nu_{\mu} \rightarrow \nu_{\mu}, \nu_{\mu} \rightarrow \nu_{\tau}$ ) contribute in the probability level in probing the questions related to $\mathrm{CPV}, \mathrm{MH}$, and the octant of $\theta_{23}$ at the DUNE baseline of $1300 \mathrm{~km}$. The $\Delta \chi^{2}$ estimation for $\mathrm{CPV}$ in Fig. 4 gives a numerical measure of the difference between the $C P$ conserving value (test $\delta=0$ or $\pi)$ and all values of true $\delta(\in[-\pi, \pi])$ and is maximum around true $\delta \approx \pm \pi / 2$. In Fig. 8 , we do a probability analysis by plotting $P_{\mu \beta}(\beta=e, \mu, \tau)$ for $\delta=-\pi / 2$ (solid black), 0 (dashed black), and their absolute difference (green). Finally we also show a $\Delta \chi^{2}$-like quantity $X$ (magenta) defined in the probability level following the statistical part of Eq. (3). For Fig. 8, the explicit definition of this quantity is

$$
X_{\mathrm{CPV}}=2\left[P_{\mu \beta}(0)-P_{\mu \beta}(-\pi / 2)+P_{\mu \beta}(-\pi / 2) \ln \frac{P_{\mu \beta}(-\pi / 2)}{P_{\mu \beta}(0)}\right],
$$

where the argument within the parentheses are the values of the $C P$ phase $\delta$. Figure 8 shows that though the magnitudes of the difference of probabilities (green) are in the similar ballpark for all the three channels, the contribution to the $\Delta \chi^{2}$-like quantity $X$ (magenta) mainly comes from the $\nu_{\mu} \rightarrow \nu_{e}$ channel. This is because the fractional difference of the probabilities (which dominates the estimation of $\Delta \chi^{2}$ ) in the $\nu_{\mu} \rightarrow \nu_{e}$ channel is much higher, owing to the small magnitudes of $P_{\mu e}$. As is clear from the insets in Fig. 8, this fractional difference (magenta) is tiny for the $\nu_{\mu} \rightarrow \nu_{\mu}$ and $\nu_{\mu} \rightarrow \nu_{\tau}$ channels.

Similarly, in Fig. 9, we analyze the contributions of the three channels in probing the MH degeneracy. For the two 

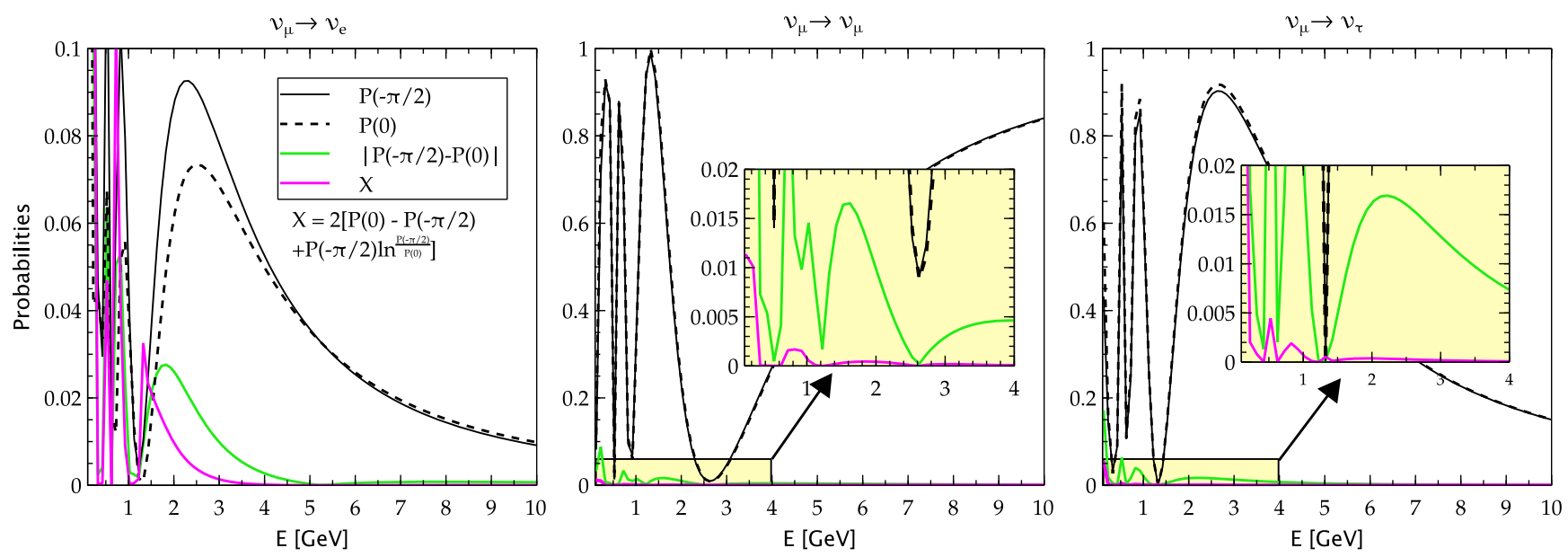

FIG. 8. Comparison of probabilities (solid and dashed black) and their differences (green) for the three channels $\nu_{\mu} \rightarrow \nu_{e}$ (first panel), $\nu_{\mu} \rightarrow \nu_{\mu}$ (second), and $\nu_{\mu} \rightarrow \nu_{\tau}$ (third). A $\Delta \chi^{2}$-like quantity $X$ (magenta) in the probability level gives a measure of the contributions of these three channels to the sensitivity to $\mathrm{CPV}$.
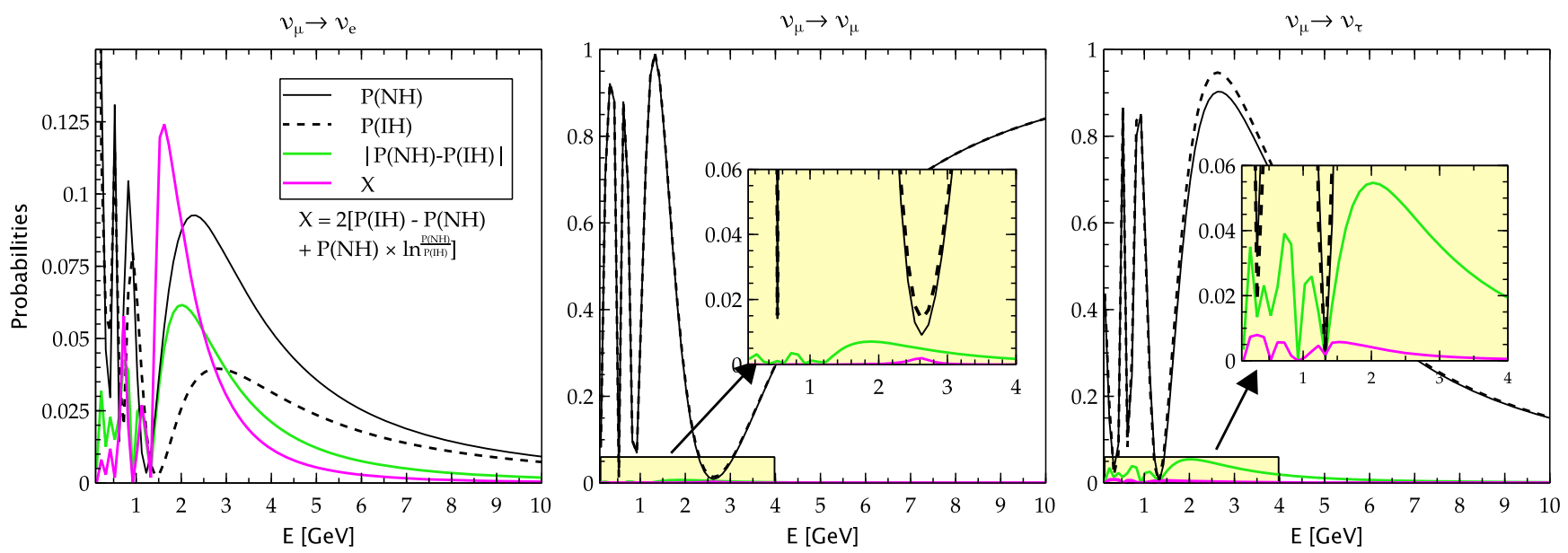

FIG. 9. Similar to Fig. 8 but for contribution to explore the MH degeneracy.

opposite hierarchies $\mathrm{NH}$ and $\mathrm{IH}$, we show $P_{\mu \beta}$ (solid black and dashed black), their difference (green), and the $\Delta \chi^{2}$ like quantity (magenta) defined below:

$X_{\mathrm{MH}}=2\left[P_{\mu \beta}(\mathrm{IH})-P_{\mu \beta}(\mathrm{NH})+P_{\mu \beta}(\mathrm{NH}) \ln \frac{P_{\mu \beta}(\mathrm{NH})}{P_{\mu \beta}(\mathrm{IH})}\right]$.

We take the $C P$ phase to be $-\pi / 2$ here. It can be easily observed that $X$ is again dominated by the $\nu_{\mu} \rightarrow \nu_{e}$ channel, while the $\nu_{\mu} \rightarrow \nu_{\mu}$ channel gives almost no contribution. Interestingly, though the difference of $P_{\mu \tau}$ for $\mathrm{NH}$ and $\mathrm{IH}$ (i.e., green curve) is very similar in magnitude with that of $P_{\mu e}$, the large value of $P_{\mu \tau}$ makes the fractional difference and consequently the value of the $\Delta \chi^{2}$-like quantity $X$ insignificant.
Finally the probability level analyses for $\theta_{23}$-octant degeneracy is illustrated in Fig. 10. The $\Delta \chi^{2}$-like quantity $X$ is defined as follows:

$X_{\mathrm{OCT}}=2\left[P_{\mu \beta}(\mathrm{LO})-P_{\mu \beta}(\mathrm{HO})+P_{\mu \beta}(\mathrm{HO}) \ln \frac{P_{\mu \beta}(\mathrm{HO})}{P_{\mu \beta}(\mathrm{LO})}\right]$,

where the value of the $C P$ phase $\delta$ was kept at its best-fit value of $-\pi / 2$. We see both the $\nu_{\mu} \rightarrow \nu_{e}$ and $\nu_{\mu} \rightarrow \nu_{\mu}$ channel contribute to the octant sensitivity, the latter slightly dominating around the crucial energy region of 2-3 GeV,

\footnotetext{
${ }^{10}$ The very small magnitude of $P_{\mu \mu}$ around this energy range helps to enhance $\Delta \chi^{2}$-like quantity $X$.
} 

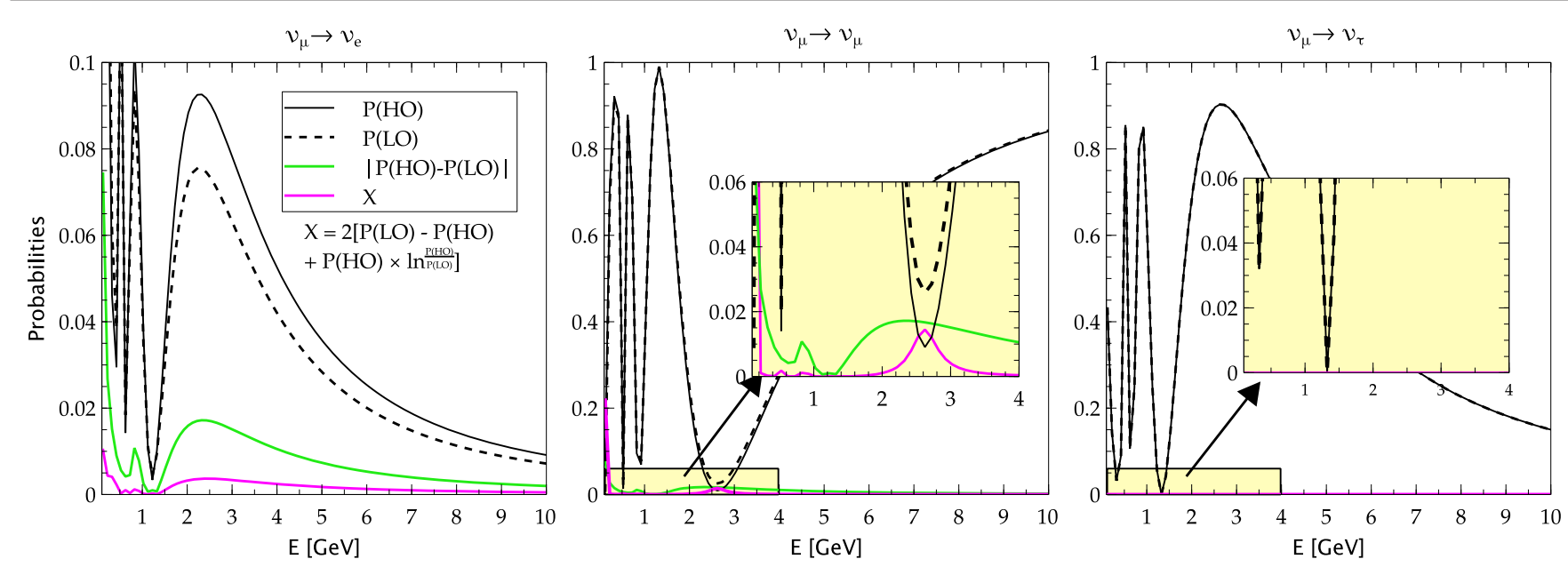

FIG. 10. Similar to Fig. 8 but for contribution to explore the $\theta_{23}$-octant degeneracy.

[1] C. Cowan, F. Reines, F. Harrison, H. Kruse, and A. McGuire, Science 124, 103 (1956).

[2] B. Pontecorvo, Sov. Phys. JETP 6, 429 (1957).

[3] B. Pontecorvo, Sov. Phys. JETP 7, 172 (1958).

[4] Z. Maki, M. Nakagawa, and S. Sakata, Prog. Theor. Phys. 28, 870 (1962).

[5] V. Gribov and B. Pontecorvo, Phys. Lett. 28B, 493 (1969).

[6] T. Kajita and A. B. McDonald, the Nobel Prize in Physics 2015, https://www.nobelprize.org/prizes/physics/2015/summary/.

[7] P. Zyla et al. (Particle Data Group), Prog. Theor. Exp. Phys. 2020, 083C01 (2020).

[8] A. Sakharov, Sov. Phys. Usp. 34, 392 (1991).

[9] M. Fukugita and T. Yanagida, Phys. Lett. B 174, 45 (1986).

[10] S. Davidson, E. Nardi, and Y. Nir, Phys. Rep. 466, 105 (2008).

[11] G. C. Branco, R. G. Felipe, and F. R. Joaquim, Rev. Mod. Phys. 84, 515 (2012).

[12] S. Petcov, Phys. Lett. 110B, 245 (1982).

[13] S. F. King, A. Merle, S. Morisi, Y. Shimizu, and M. Tanimoto, New J. Phys. 16, 045018 (2014).

[14] W. Haxton and G. Stephenson, Prog. Part. Nucl. Phys. 12, 409 (1984).

[15] S. R. Elliott and J. Engel, J. Phys. G 30, R183 (2004).

[16] C. Aalseth et al., arXiv:hep-ph/0412300.

[17] C. Lam, Phys. Lett. B 507, 214 (2001).

[18] P. Harrison and W. Scott, Phys. Lett. B 547, 219 (2002).

[19] Z.-Z. Xing and Z.-H. Zhao, Rep. Prog. Phys. 79, 076201 (2016).

[20] K. Abe et al. (T2K Collaboration), Phys. Rev. Lett. 112, 061802 (2014).

[21] D. S. Ayres et al. (NOvA Collaboration), arXiv:hep-ex/ 0503053.

[22] K. Abe et al. (T2K Collaboration), Nature (London) 580, 339 (2020); 583, E16 (2020).

[23] M. A. Acero et al. (NOvA Collaboration), Phys. Rev. Lett. 123, 151803 (2019).
[24] P. de Salas, D. Forero, S. Gariazzo, P. Martínez-Miravé, O. Mena, C. Ternes, M. Tórtola, and J. Valle, arXiv:2006 .11237.

[25] Valencia-Globalfit, http://globalfit.astroparticles.es/ (2020).

[26] F. Capozzi, E. Lisi, A. Marrone, and A. Palazzo, Prog. Part. Nucl. Phys. 102, 48 (2018).

[27] I. Esteban, M. C. Gonzalez-Garcia, A. HernandezCabezudo, M. Maltoni, and T. Schwetz, J. High Energy Phys. 01 (2019) 106.

[28] M. A. Tórtola, G. A. Barenboim, and C. A. Ternes, J. High Energy Phys. 07 (2020) 155.

[29] K. J. Kelly, P. A. Machado, S. J. Parke, Y. F. Perez Gonzalez, and R. Zukanovich-Funchal, arXiv:2007.08526.

[30] R. Acciarri et al. (DUNE Collaboration), arXiv:1512.06148.

[31] B. Abi et al. (DUNE Collaboration), arXiv:2002.03005.

[32] B. Abi et al. (DUNE Collaboration), Eur. Phys. J. C 80, 978 (2020).

[33] M. Masud, M. Bishai, and P. Mehta, Sci. Rep. 9, 352 (2019).

[34] M. Masud, S. Roy, and P. Mehta, Phys. Rev. D 99, 115032 (2019).

[35] A. De Gouvêa, K. J. Kelly, G. Stenico, and P. Pasquini, Phys. Rev. D 100, 016004 (2019).

[36] A. Ghoshal, A. Giarnetti, and D. Meloni, J. High Energy Phys. 12 (2019) 126.

[37] P. Huber, M. Lindner, T. Schwetz, and W. Winter, J. High Energy Phys. 11 (2009) 044.

[38] S. K. Agarwalla, S. Prakash, and S. Sankar, J. High Energy Phys. 07 (2013) 131.

[39] P. Machado, H. Minakata, H. Nunokawa, and R. Zukanovich Funchal, J. High Energy Phys. 05 (2014) 109.

[40] M. Ghosh, S. Goswami, and S. K. Raut, Mod. Phys. Lett. A 32, 1750034 (2017).

[41] M. Ghosh, P. Ghoshal, S. Goswami, N. Nath, and S. K. Raut, Phys. Rev. D 93, 013013 (2016).

[42] M. Ghosh, S. Goswami, and S. K. Raut, Eur. Phys. J. C 76, 114 (2016). 
[43] N. Nath, M. Ghosh, and S. Goswami, Nucl. Phys. B913, 381 (2016).

[44] P. Coloma, H. Minakata, and S. J. Parke, Phys. Rev. D 90, 093003 (2014).

[45] C. Adams et al. (LBNE Collaboration), arXiv:1307.7335.

[46] P. Ballett, S. F. King, S. Pascoli, N. W. Prouse, and T. Wang, Phys. Rev. D 96, 033003 (2017).

[47] DUNE Internal Document DUNE-doc-20412-v1.

[48] P. Huber, M. Lindner, and W. Winter, Comput. Phys. Commun. 167, 195 (2005).

[49] P. Huber, J. Kopp, M. Lindner, M. Rolinec, and W. Winter, Comput. Phys. Commun. 177, 432 (2007).

[50] A. M. Dziewonski and D. L. Anderson, Phys. Earth Planet. Interiors 25, 297 (1981).

[51] R. Gandhi, P. Ghoshal, S. Goswami, P. Mehta, and S. U. Sankar, Phys. Rev. Lett. 94, 051801 (2005).

[52] R. Gandhi, P. Ghoshal, S. Goswami, P. Mehta, and S. U. Sankar, Phys. Rev. D 73, 053001 (2006).

[53] K. J. Kelly and S. J. Parke, Phys. Rev. D 98, 015025 (2018).
[54] A. Chatterjee, F. Kamiya, C. A. Moura, and J. Yu, arXiv: 1809.09313.

[55] T. Alion et al. (DUNE Collaboration), arXiv:1606.09550.

[56] S. Agostinelli et al. (GEANT4 Collaboration), Nucl. Instrum. Methods Phys. Res., Sect. A 506, 250 (2003).

[57] J. Allison et al., IEEE Trans. Nucl. Sci. 53, 270 (2006).

[58] P. Huber, M. Lindner, and W. Winter, Nucl. Phys. B645, 3 (2002).

[59] G. L. Fogli, E. Lisi, A. Marrone, D. Montanino, and A. Palazzo, Phys. Rev. D 66, 053010 (2002).

[60] M. Gonzalez-Garcia and M. Maltoni, Phys. Rev. D 70, 033010 (2004).

[61] R. Gandhi, P. Ghoshal, S. Goswami, P. Mehta, S. U. Sankar, and S. Shalgar, Phys. Rev. D 76, 073012 (2007).

[62] X. Qian, A. Tan, W. Wang, J. J. Ling, R. D. McKeown, and C. Zhang, Phys. Rev. D 86, 113011 (2012).

[63] M. Masud, A. Chatterjee, and P. Mehta, J. Phys. G 43, 095005 (2016).

[64] M. Masud and P. Mehta, Phys. Rev. D 94, 013014 (2016).

[65] M. Masud and P. Mehta, Phys. Rev. D 94, 053007 (2016). 